\title{
A simplified ordinal analysis of first-order reflection
}

\author{
Toshiyasu Arai \\ Graduate School of Science, Chiba University \\ 1-33, Yayoi-cho, Inage-ku, Chiba, 263-8522, JAPAN \\ tosarai@faculty.chiba-u.jp *
}

\begin{abstract}
In this note we give a simplified ordinal analysis of first-order reflection. An ordinal notation system $O T$ is introduced based on $\psi$-functions. Provable $\Sigma_{1}$-sentences on $L_{\omega_{1}^{C K}}$ are bounded through cut-elimination on operator controlled derivations.
\end{abstract}

\section{Introduction}

Let $O R D$ denote the class of all ordinals, $A \subset O R D$ and $\alpha$ a limit ordinal. $\alpha$ is said to be $\Pi_{n}$-reflecting on $A$ iff for any $\Pi_{n}$-formula $\phi(x)$ and any $b \in L_{\alpha}$, if $\left\langle L_{\alpha}, \in\right\rangle \models \phi(b)$, then there exists a $\beta \in A \cap \alpha$ such that $b \in L_{\beta}$ and $\left\langle L_{\beta}, \in\right\rangle \models$ $\phi(b)$. Let us write $\alpha \in r M_{n}(A): \Leftrightarrow \alpha$ is $\Pi_{n}$-reflecting on $A$. Also $\alpha$ is said to be $\Pi_{n}$-reflecting iff $\alpha$ is $\Pi_{n}$-reflecting on $O R D$.

It is not hard for us to show that the assumption that the universe is $\Pi_{n^{-}}$ reflecting is proof-theoretically reducible to iterabilities of the lower operation $r M_{n-1}$ (and Mostowski collapsings), cf. [3].

In this paper we aim an ordinal analysis of $\Pi_{n}$-reflection. Such an analysis was done by Pohlers and Stegert [7] using reflection configurations introduced in M. Rathjen [9, and an alternative analysis in [1,2, 4] with the complicated combinatorial arguments of ordinal diagrams and finite proof figures. Our approach is simpler in view of combinatorial arguments. In [1, a $\Pi_{n}$-reflecting universe is resolved in ramified hierarchies of lower Mahlo operations, and ultimately in iterations of recursively Mahlo operations. Our ramification process is akin to a tower, i.e., has an exponential structure. It is natural that an exponential structure emerges in lowering and eliminating first-order formulas (in reflections), cf. ordinal analysis for the fragments I $\Sigma_{n-3}$ of the first-order arithmetic. Mahlo classes $M h_{k}(\xi)$ defined in Definition 2.5 to resolve or approximate

\footnotetext{
* Current address: Graduate School of Mathematical Sciences, The University of Tokyo, 3-8-1 Komaba, Meguro-ku, Tokyo 153-8914, JAPAN tosarai@ms.u-tokyo.ac.jp
} 
$\Pi_{n}$-reflection are based on similar structure. As in Rathjen's analysis for $\Pi_{3^{-}}$ reflection in [8, thinning operations are applied on the Mahlo classes $M h_{k}(\xi)$, and this yields an exponential structure similar to the one in [1] as follows.

Let us consider the simplest case $N=4$. Let $\Lambda:=\varepsilon_{\mathbb{K}+1}$, the next epsilon number above the lease $\Pi_{4}$-reflecting ordinal $\mathbb{K}$. Roughly $\pi \in M h_{3}(\xi)$ designates the fact that an ordinal $\pi$ is $\Pi_{3}$-reflecting on $M h_{3}(\nu)$ for any $\nu<\xi<\Lambda$. Suppose a $\Pi_{3}$-sentence $\theta$ on $L_{\pi}$ is derived from the assumption $\pi \in M h_{3}(\xi)$. We need to find an ordinal $\kappa<\pi$ for which $L_{\kappa} \models \theta$ holds. It turns out that $\kappa \in M h_{2}\left(\Lambda^{\xi} a\right)$ suffices for an ordinal $a<\Lambda$, where the ordinal $\kappa$ in the class $M h_{2}\left(\Lambda^{\xi} a\right)$ is $\Pi_{2}$-reflecting on classes $M h_{2}\left(\Lambda^{\xi} b\right) \cap M h_{3}(\nu)$ for any $b<a$ and any $\nu<\xi$. Note that the class $M h_{2}\left(\Lambda^{\xi} a\right)$ is not obtained through iterations of recursively Mahlo operations since it involves $\Pi_{4}$-definable classes $M h_{3}(\nu)$. The classes $M h_{3}(\nu)(\nu<\xi)$ for the assumption $\pi \in M h_{3}(\xi)$ are thinned out with the new classes $M h_{2}\left(\Lambda^{\xi} b\right)(b<\Lambda)$, cf. Lemma 5.1 .

Our theorem runs as follows. Let $\mathrm{KP} \Pi_{N}$ denote the set theory for $\Pi_{N}$-reflecting universes, and $\mathrm{KP} \omega$ the Kripke-Platek set theory with the axiom of infinity. OT is a computable notation system of ordinals defined in section $3, \Omega=\omega_{1}^{C K}$ and $\psi_{\Omega}$ is a collapsing function such that $\psi_{\Omega}(\alpha)<\Omega$. $\mathbb{K}$ is an ordinal term denoting the least $\Pi_{N}$-reflecting ordinal in the theorems.

Theorem 1.1 Suppose $\mathrm{KP}_{N} \vdash \theta$ for a $\Sigma_{1}(\Omega)$-sentence $\theta$. Then we can find an $n<\omega$ such that for $\alpha=\psi_{\Omega}\left(\omega_{n}(\mathbb{K}+1)\right), L_{\alpha} \models \theta$.

Actually the bound is seen to be tight, cf. [5].

Theorem 1.2 $\mathrm{KP}_{N}$ proves that each initial segment $\left\{\alpha \in O T: \alpha<\psi_{\Omega}\left(\omega_{n}(\mathbb{K}+1)\right)\right\}(n=1,2, \ldots)$ is well-founded.

Thus the ordinal $\psi_{\Omega}\left(\varepsilon_{\mathbb{K}+1}\right)$ is seen to be the proof-theoretic ordinal of $\mathrm{KP} \Pi_{N}$.

\section{Theorem 1.3}

$$
\psi_{\Omega}\left(\varepsilon_{\mathbb{K}+1}\right)=\left|\mathrm{KP} \Pi_{N}\right|_{\Sigma_{1}^{\Omega}}:=\min \left\{\alpha \leq \omega_{1}^{C K}: \forall \theta \in \Sigma_{1}\left(\mathrm{KP}_{N} \vdash \theta^{L_{\Omega}} \Rightarrow L_{\alpha} \models \theta\right)\right\} .
$$

$A \subset O R D$ is $\Pi_{n}^{1}$-indescribable in $\alpha$ iff for any $\Pi_{n}^{1}$-formula $\phi(X)$ and any $B \subset O R D$, if $\left\langle L_{\alpha}, \in ; B \cap \alpha\right\rangle \models \phi(B \cap \alpha)$, then there exists a $\beta \in A \cap \alpha$ such that $\left\langle L_{\beta}, \in ; B \cap \beta\right\rangle \models \phi(B \cap \beta)$. A regular cardinal $\pi$ is $\Pi_{n}^{1}$-indescribable ifff $O R D$ is $\Pi_{n}^{1}$-indescribable in $\pi$.

Let us mention the contents of this paper. In the next section 2 we define simultaneously iterated Skolem hulls $\mathcal{H}_{\alpha}(X)$ of sets $X$ of ordinals, ordinals $\psi_{\kappa}^{\vec{\xi}}(\alpha)$ for regular cardinals $\kappa, \alpha<\varepsilon_{\mathbb{K}+1}$ and sequences $\vec{\xi}=\left(\xi_{2}, \ldots, \xi_{N-1}\right)$ of ordinals $\xi_{i}<\varepsilon_{\mathbb{K}+2}$, and classes $M h_{k}^{\alpha}(\xi)$ under the assumption that a $\Pi_{N-2}^{1}$-indescribable cardinal $\mathbb{K}$ exists. It is shown that for $2 \leq k<N, \alpha<\varepsilon_{\mathbb{K}+1}$ and each $\xi<\varepsilon_{\mathbb{K}+2}$, $\left(\mathbb{K}\right.$ is a $\Pi_{N-2}^{1}$-indescribable cardinal $) \rightarrow \mathbb{K} \in M h_{k}^{\alpha}(\xi)$ in $\mathrm{ZF}+(V=L)$.

In section 3 a computable notation system $O T$ of ordinals is extracted. Following W. Buchholz [6], operator controlled derivations for $\mathrm{KP}_{N}$ is introduced 
in section 4, and inference rules for $\Pi_{N}$-reflection are eliminated from derivations in section 5 This completes a proof of Theorem 1.1 for an upper bound.

IH denotes the Induction Hypothesis, MIH the Main IH and SIH the Subsidiary IH. We are assuming tacitly the axiom of constructibility $V=L$. Throughout of this paper $N \geq 3$ is a fixed integer.

\section{Ordinals for $\Pi_{N}$-reflection}

In this section we work in the set theory $\mathrm{ZFLK}_{N}$ obtained from ZFL $=\mathrm{ZF}+(V=$ $L$ ) by adding the axiom $\exists \mathbb{K}\left(\mathbb{K}\right.$ is $\Pi_{N-2}^{1}$-indescribable) for a fixed integer $N \geq 3$. For ordinals $\alpha, \varepsilon(\alpha)$ denotes the least epsilon number above $\alpha$.

Let $O R D \subset V$ denote the class of ordinals, $\mathbb{K}$ the least $\Pi_{N-2}^{1}$-indescribable cardinal, and Reg the set of regular ordinals below $\mathbb{K}$. $\Theta$ denotes finite sets of ordinals $\leq \mathbb{K} . u, v, w, x, y, z, \ldots$ range over sets in the universe, $a, b, c, \alpha, \beta, \gamma, \ldots$ range over ordinals $<\Lambda, \xi, \zeta, \nu, \mu, \iota, \ldots$ range over ordinals $<\varepsilon(\Lambda)=\varepsilon_{\mathbb{K}+2}$, $\vec{\xi}, \vec{\zeta}, \vec{\nu}, \vec{\mu}, \vec{\imath}, \ldots$ range over finite sequences over ordinals $<\varepsilon(\Lambda)$, and $\pi, \kappa, \rho, \sigma, \tau, \lambda, \ldots$ range over regular ordinals. $\theta$ denotes formulas.

Let $\vec{\xi}=\left(\xi_{0}, \ldots, \xi_{m-1}\right)$ be a sequence of ordinals. The length $\operatorname{lh}(\vec{\xi}):=m$. Sequences consisting of a single element $(\xi)$ is identified with the ordinal $\xi$, and $\emptyset$ denotes the empty sequence. $\overrightarrow{0}$ denotes ambiguously a zero-sequence $(0, \ldots, 0)$ with its length $0 \leq \operatorname{lh}(\overrightarrow{0}) \leq N-1 . \vec{\xi} * \vec{\mu}=\left(\xi_{0}, \ldots, \xi_{m-1}\right) *\left(\mu_{0}, \ldots, \nu_{n-1}\right)=$ $\left(\xi_{0}, \ldots, \xi_{m-1}, \mu_{0}, \ldots, \mu_{n-1}\right)$ denotes the concatenated sequence of $\vec{\xi}$ and $\vec{\mu}$.

$\Lambda=\varepsilon(\mathbb{K})=\varepsilon_{\mathbb{K}+1}$ denotes the next epsilon number above the least $\Pi_{N-2^{-}}$ indescribable cardinal $\mathbb{K}$, and $\varepsilon(\Lambda)=\varepsilon_{\mathbb{K}+2}$ the next epsilon number above $\Lambda$.

Definition 2.1 For a non-zero ordinal $\xi<\varepsilon(\Lambda)$, its Cantor normal form with base $\Lambda$ is uniquely determined as

$$
\xi={ }_{N F} \sum_{i \leq m} \Lambda^{\xi_{i}} a_{i}=\Lambda^{\xi_{m}} a_{m}+\cdots+\Lambda^{\xi_{0}} a_{0}
$$

where $\xi_{m}>\cdots>\xi_{0}, 0<a_{i}<\Lambda$.

1. $K(\xi)=\left\{a_{i}: i \leq m\right\} \cup \bigcup\left\{K\left(\xi_{i}\right): i \leq m\right\}$ is the set of components of $\xi$ with $K(0)=\emptyset$. For a sequence $\vec{\xi}=\left(\xi_{0}, \ldots, \xi_{n-1}\right)$ of ordinals $\xi_{i}<\varepsilon(\Lambda)$, $K(\vec{\xi}):=\bigcup\left\{K\left(\xi_{i}\right): i<n\right\}$.

2. For $\xi>1, t e(\xi)=\xi_{0}$ in (11) is the tail exponent, and $h e(\xi)=\xi_{m}$ is the head exponent of $\xi$, resp. The head $H d(\xi):=\Lambda^{\xi_{m}} a_{m}$, and the tail $T l(\xi):=\Lambda^{\xi_{0}} a_{0}$ of $\xi$.

3. $h e^{(i)}(\xi)$ is the $i$-th head exponent of $\xi$, defined recursively by $h e^{(0)}(\xi)=\xi, h e^{(i+1)}(\xi)=h e\left(h e^{(i)}(\xi)\right)$.

The $i$-th tail exponent te $e^{(i)}(\xi)$ is defined similarly. 
4. $\zeta$ is a part of $\xi$, denoted by $\zeta \leq_{p t} \xi$ iff

$\zeta={ }_{N F} \sum_{i \geq n} \Lambda^{\xi_{i}} a_{i}=\Lambda^{\xi_{m}} a_{m}+\cdots+\Lambda^{\xi_{n}} a_{n}$ for an $n(0 \leq n \leq m+1)$.

$\zeta<_{p t} \xi: \Leftrightarrow \zeta \leq_{p t} \xi \& \zeta \neq \xi$.

5. A sequence $\vec{\mu}=\left(\mu_{0}, \ldots, \mu_{n}\right)$ is an iterated tail parts of $\xi$, denoted by $\vec{\mu} \subset_{p t} \xi$ iff $\mu_{0} \leq_{p t} \xi \& \forall i<n\left(\mu_{i+1} \leq_{p t} t e\left(\mu_{i}\right)\right)$.

6. $\vec{\nu}=\left(\nu_{0}, \ldots, \nu_{n}\right) * \overrightarrow{0}<\xi$ iff there exists a sequence $\vec{\mu}=\left(\mu_{0}, \ldots, \mu_{n}\right)$ such that $\vec{\mu} \subset_{p t} \xi$ and $\nu_{i}<\mu_{i}$ for every $i \leq n$.

7. Let $\vec{\nu}=\left(\nu_{0}, \ldots, \nu_{n}\right)$ and $\vec{\xi}=\left(\xi_{0}, \ldots, \xi_{n}\right)$ be sequences of ordinals in the same length, and $0 \leq k \leq n$.

$\vec{\nu}<_{k} \vec{\xi}: \Leftrightarrow \forall i<k\left(\nu_{i} \leq \xi_{i}\right) \wedge\left(\nu_{k}, \ldots, \nu_{n}\right)<\xi_{k}$.

8. $\zeta$ is a step-down of $\xi$, denoted by $\zeta<_{s d} \xi$ iff

$\zeta=\Lambda^{\xi_{m}} a_{m}+\cdots+\Lambda^{\xi_{1}} a_{1}+\Lambda^{\xi_{0}} b+\nu$ for some ordinals $b<a_{0}$ and $\nu<\Lambda^{\xi_{0}}$.

9. $\vec{\nu}=\left(\nu_{0}, \ldots, \nu_{n}\right) * \overrightarrow{0}<_{s d} \xi$ iff $\nu_{i}<_{s d} t e^{(i)}(\xi)$ for every $i \leq n$.

10. $\zeta \leq_{s p} \xi: \Leftrightarrow \exists \mu \leq_{p t} \xi\left(\zeta \leq_{s d} \mu\right)$, and $\zeta<_{s p} \xi: \Leftrightarrow \exists \mu \leq_{p t} \xi\left(\zeta<_{s d} \mu\right)$.

11. $\vec{\nu}<_{s p} \xi$ iff $\vec{\nu}<_{s d} \mu$ for a $\mu \leq_{p t} \xi$.

Let $p(\vec{\nu}, \xi)$ denote the number $p(0 \leq p<m)$ such that $\xi={ }_{N F} \mu+$ $\sum_{i<p} \Lambda^{\xi_{i}} a_{i}$ for $\mu=\Lambda^{\xi_{m}} a_{m}+\cdots+\Lambda^{\xi_{p}} a_{p}$ and $\vec{\nu}<_{s d} \mu$.

Note that $(\nu) * \overrightarrow{0}<\xi \Leftrightarrow \nu<\xi$, and $\left(\xi, t e(\xi), t e^{(2)}(\xi), \ldots\right) \subset_{p t} \xi$. Also $\zeta<_{s d} \xi \Leftrightarrow \zeta<\xi$ if $\xi<\Lambda$.

Proposition $2.2 \xi<\mu<\varepsilon(\Lambda) \Rightarrow t e(\xi) \leq h e(\xi) \leq h e(\mu)$.

Proposition 2.3 $\vec{\nu}<\xi \leq \zeta \Rightarrow \vec{\nu}<\zeta$.

Proof by induction on the lengths $n=\operatorname{lh}(\vec{\nu})$. Let $\vec{\mu}=\left(\mu_{0}, \ldots, \mu_{n-1}\right)$ be a sequence for $\vec{\nu}=\left(\nu_{0}, \ldots, \nu_{n-1}\right)$ such that $\vec{\mu} \subset p t \xi$ and $\forall i \leq n-1\left(\nu_{i}<\mu_{i}\right)$, cf. Definition 2.16]

If $n=1$, then $\nu_{0}<\mu_{0} \leq_{p t} \xi \leq \zeta . \nu_{0}<\zeta \leq_{p t} \zeta$ yields $\vec{\nu}=\left(\nu_{0}\right)<\zeta$.

Let $n>1$. We have $\left(\nu_{1}, \ldots, \nu_{n-1}\right)<t e\left(\mu_{0}\right)$ with $\left(\mu_{1}, \ldots, \mu_{n-1}\right) \subset_{p t} t e\left(\mu_{0}\right)$. We show the existence of a $\lambda$ such that $\mu_{0} \leq \lambda \leq{ }_{p t} \zeta$ and $t e\left(\mu_{0}\right) \leq t e(\lambda)$. Then IH yields $\left(\nu_{1}, \ldots, \nu_{n-1}\right)<t e(\lambda)$, and $\vec{\nu}<\zeta$ follows.

If $\mu_{0} \leq_{p t} \zeta$, then $\lambda=\mu_{0}$ works. Suppose $\mu_{0} \mathbb{E}_{p t} \zeta$. On the other hand we have $\mu_{0} \leq_{p t} \xi \leq \zeta$. This means that $\xi<\zeta$ and there exists a $\lambda \leq_{p t} \zeta$ such that $\mu_{0}<\lambda$ and $t e\left(\mu_{0}\right) \leq t e(\lambda)$. 


\section{$2.1 \quad$ Ordinals}

Definition 2.4 1. For $i<\omega$ and $\xi<\varepsilon(\Lambda), \Lambda_{i}(\xi)$ is defined recursively by $\Lambda_{0}(\xi)=\xi$ and $\Lambda_{i+1}(\xi)=\Lambda^{\Lambda_{i}(\xi)}$.

2. For $A \subset O R D$, limit ordinals $\alpha$ and $i \geq 0$, let $\alpha \in M_{2+i}(A)$ iff $A \cap$ $\alpha$ is $\Pi_{i}^{1}$-indescribable in $\alpha$.

3. $\kappa^{+}$denotes the next regular ordinal above $\kappa$.

4. $\Omega_{\alpha}:=\omega_{\alpha}$ for $\alpha>0, \Omega_{0}:=0$, and $\Omega=\Omega_{1}$.

Define simultaneously classes $\mathcal{H}_{\alpha}(X), M h_{k}^{\alpha}(\xi)$, and ordinals $\psi_{\kappa}^{\vec{\xi}}(\alpha)$ as follows. We see that these are $\Sigma_{1}$-definable as a fixed point in ZFL, cf. Proposition 2.7.

Let $a<\Lambda$, and $\varphi$ denote the binary Veblen function. Let us define a Skolem hull $\mathcal{H}_{a}(X)$ of $\{0, \mathbb{K}\} \cup X$ under the functions $+, \alpha \mapsto \omega^{\alpha},(\alpha, \beta) \mapsto \varphi \alpha \beta(\alpha, \beta<$ $\mathbb{K}), \alpha \mapsto \Omega_{\alpha}(\alpha<\mathbb{K})$ and $\psi$-functions. Reg denotes the set of regular ordinals $\leq$ $\mathbb{K}$.

Definition 2.5 $\mathcal{H}_{a}[Y](X):=\mathcal{H}_{a}(Y \cup X)$ for sets $Y \subset \mathbb{K}$.

1. (Inductive definition of $\mathcal{H}_{a}(X)$ ).

(a) $\{0, \mathbb{K}\} \cup X \subset \mathcal{H}_{a}(X)$.

(b) $x, y \in \mathcal{H}_{a}(X) \Rightarrow x+y \in \mathcal{H}_{a}(X), x \in \mathcal{H}_{a}(X) \Rightarrow \omega^{x} \in \mathcal{H}_{a}(X)$, and $x, y \in \mathcal{H}_{a}(X) \cap \mathbb{K} \Rightarrow \varphi x y \in \mathcal{H}_{a}(X)$.

(c) $\mathbb{K}>\alpha \in \mathcal{H}_{a}(X) \Rightarrow \Omega_{\alpha} \in \mathcal{H}_{a}(X)$.

(d) If $\pi \in \mathcal{H}_{a}(X) \cap \operatorname{Reg}$ and $b \in \mathcal{H}_{a}(X) \cap a$, then $\psi_{\pi}(b) \in \mathcal{H}_{a}(X)$.

(e) If $\{b, \xi\} \subset \mathcal{H}_{a}(X)$ with $\xi \leq b<a$, then $\kappa=\psi_{\mathbb{K}}^{\overrightarrow{0} *(\xi)}(b) \in \mathcal{H}_{a}(X)$, where $\operatorname{lh}(\overrightarrow{0})=N-3$.

(f) Let $\{\pi, b, c\} \subset \mathcal{H}_{a}(X)$ with $\pi<\mathbb{K}, 2 \leq k<N-1$ an integer, and $\vec{\xi}=\left(\xi_{2}, \ldots, \xi_{k}, \xi_{k+1}\right) * \overrightarrow{0}$ a sequence of ordinals $\xi_{i}<\varepsilon(\Lambda)$ with $\operatorname{lh}(\overrightarrow{0})=N-2-k$ such that $\xi_{k+1} \neq 0$ and $K(\vec{\xi}) \subset \mathcal{H}_{a}(X)$. Assume $\max (K(\vec{\xi}) \cup\{c\}) \leq b<a$, and $\pi \in M h_{2}^{b}(\vec{\xi})$.

Then $\kappa=\psi_{\pi}^{\vec{\nu}}(b) \in \mathcal{H}_{a}(X)$ for the sequence $\vec{\nu}=\left(\xi_{2}, \ldots, \xi_{k}+\Lambda^{\xi_{k+1}} c\right) * \overrightarrow{0}$ with $\operatorname{lh}(\overrightarrow{0})=N-1-k$.

(g) Let $\{\pi, b\} \subset \mathcal{H}_{a}(X)$ with $\pi<\mathbb{K}$, and $0 \neq \xi<\varepsilon(\Lambda)$ an ordinal with $K(\xi) \subset \mathcal{H}_{a}(X)$. Let $\vec{\nu}=\left(\nu_{2}, \ldots, \nu_{N-1}\right)$ be a sequence of ordinals $<$ $\varepsilon(\Lambda)$ such that $K(\vec{\nu}) \subset \mathcal{H}_{a}(X)$. Assume $\max K(\vec{\nu}) \leq b<a, K(\vec{\nu}) \subset$ $\mathcal{H}_{b}(\pi), \pi \in M h_{2}^{b}(\xi)$, and $\vec{\nu}<\xi$, cf. Definition 2.16]

Then $\kappa=\psi_{\pi}^{\vec{\nu}}(b) \in \mathcal{H}_{a}(X)$.

2. (Definitions of $M h_{k}^{a}(\xi)$ and $M h_{k}^{a}(\vec{\xi})$ )

First let $\mathbb{K} \in M h_{N}^{a}(0): \Leftrightarrow \mathbb{K} \in M_{N} \Leftrightarrow \mathbb{K}$ is $\Pi_{N-2}^{1}$-indescribable. 
The classes $M h_{k}^{a}(\xi)$ are defined for $2 \leq k<N$, and ordinals $a<\Lambda$, $\xi<\varepsilon(\Lambda)$. Let $\pi$ be a regular ordinal $\leq \mathbb{K}$. Then for $\xi>0$

$$
\begin{aligned}
& \pi \in M h_{k}^{a}(\xi): \Leftrightarrow\{\pi, a\} \cup K(\xi) \subset \mathcal{H}_{a}(\pi) \& \\
& \forall \vec{\nu}<\xi\left(K(\vec{\nu}) \subset \mathcal{H}_{a}(\pi) \Rightarrow \pi \in M_{k}\left(M h_{k}^{a}(\vec{\nu})\right)\right)
\end{aligned}
$$

where $\vec{\nu}=\left(\nu_{k}, \ldots, \nu_{n}\right)(2 \leq k \leq n \leq N-1)$ varies through non-empty sequences of ordinals $<\varepsilon(\Lambda)$ and

$$
\pi \in M h_{k}^{a}(\vec{\nu}): \Leftrightarrow \pi \in \bigcap_{k \leq i \leq n} M h_{i}^{a}\left(\nu_{i}\right)
$$

By convention, let for $2 \leq k<N, \pi \in M h_{k}^{a}(0): \Leftrightarrow \pi \in M h_{2}^{a}(\emptyset): \Leftrightarrow$ $\pi$ is a limit ordinal. Note that by letting $\vec{\nu}=(0), \pi \in M h_{k}^{a}(\xi) \Rightarrow \pi \in M_{k}$ for $\xi>0$. Also $\overrightarrow{0}<1$, and $M h_{k}^{a}(1)=M_{k}$.

3. (Definition of $\psi_{\pi}^{\vec{\xi}}(a)$ )

Let $a<\Lambda$ be an ordinal, $\pi \leq \mathbb{K}$ a regular ordinal and $\vec{\xi}$ a sequence of ordinals $<\varepsilon(\Lambda)$ such that $\operatorname{lh}(\vec{\xi})=N-2$. Then let

$\psi_{\pi}^{\vec{\xi}}(a):=\min \left(\{\pi\} \cup\left\{\kappa \in M h_{2}^{a}(\vec{\xi}) \cap \pi: \mathcal{H}_{a}(\kappa) \cap \pi \subset \kappa, K(\vec{\xi}) \cup\{\pi, a\} \subset \mathcal{H}_{a}(\kappa)\right\}\right)$

Let $\psi_{\pi} a:=\psi_{\pi}^{\overrightarrow{0}} a$, where $\operatorname{lh}(\overrightarrow{0})=N-2, M h_{2}^{a}(\overrightarrow{0})=L i m$, and $\pi \in M_{2}$, i.e., $\pi$ is a regular ordinal.

Note that $\pi \in M h_{k}^{a}(\xi) \Rightarrow \forall \nu<\xi\left(\pi \in M_{k}\left(M h_{k}^{a}(\nu)\right)\right)$, since $(\nu)<\xi$ holds with $(\xi) \subset_{p t} \xi$ for $\nu<\xi$.

Proposition 2.6 $b+c \in \mathcal{H}_{a}[\Theta](d) \Rightarrow c \in \mathcal{H}_{a}[\Theta](d)$, and $\omega^{c} \in \mathcal{H}_{a}[\Theta](d) \Rightarrow c \in$ $\mathcal{H}_{a}[\Theta](d)$.

The following Proposition 2.7 is easy to see.

Proposition 2.7 Each of $x=\mathcal{H}_{a}(y)(a<\Lambda, y<\mathbb{K}), x=\psi_{\kappa} a, x \in M h_{k}^{a}(\xi)$ and $x=\psi_{\kappa}^{\vec{\xi}}(a)$, is a $\Sigma_{1}$-predicate as fixed points in ZFL.

Proof. This is seen from the facts that there exists a universal $\Pi_{n}^{1}$-formula, and by using it, $\alpha \in M_{n}(x)$ iff $\left\langle L_{\alpha}, \in\right\rangle \models m_{n}\left(x \cap L_{\alpha}\right)$ for some $\Pi_{n+1}^{1}$-formula $m_{n}(R)$ with a unary predicate $R$.

Let $A(a)$ denote the conjunction of $\forall u<\mathbb{K} \exists ! x\left[x=\mathcal{H}_{a}(u)\right]$, and $\forall \vec{\xi} \forall x\left(\max K(\vec{\xi}) \leq a \& K(\vec{\xi}) \cup\{\kappa, a\} \subset x=\mathcal{H}_{a}(\kappa) \rightarrow \exists ! b \leq \kappa\left(b=\psi_{\kappa}^{\vec{\xi}}(a)\right)\right)$, where $\operatorname{lh}(\vec{\xi})=N-2$.

Since the cardinality of the set $\mathcal{H}_{\varepsilon_{\mathbb{K}+1}}(\pi)$ is $\pi$ for any infinite cardinal $\pi \leq$ $\mathbb{K}$, pick an injection $f: \mathcal{H}_{\Lambda}(\mathbb{K}) \rightarrow \mathbb{K}$ so that $f^{\prime \prime} \mathcal{H}_{\Lambda}(\pi) \subset \pi$ for any weakly inaccessibles $\pi \leq \mathbb{K}$. 
Lemma 2.8 1. $\forall a<\Lambda A(a)$.

2. $\pi \in M h_{k}^{a}(\xi)$ is a $\Pi_{k-1}^{1}$-class on $L_{\pi}$ uniformly for weakly inaccessible cardinals $\pi \leq \mathbb{K}$ and $a, \xi$. This means that for each $k$ there exists $a \Pi_{k-1}^{1}$ formula $m h_{k}^{a}(x)$ such that $\pi \in M h_{k}^{a}(\xi)$ iff $L_{\pi} \models m h_{k}^{a}(\xi)$ for any weakly inaccessible cardinals $\pi \leq \mathbb{K}$ with $f^{\prime \prime}(\{a\} \cup K(\xi)) \subset L_{\pi}$.

3. $\mathbb{K} \in M h_{N-1}^{\alpha}(\Lambda) \cap M_{N-1}\left(M h_{N-1}^{\alpha}(\Lambda)\right)$.

\section{Proof}

2.811 We show that $A(a)$ is progressive, i.e., $\forall a<\Lambda[\forall c<a A(c) \rightarrow A(a)]$.

Assume $\forall c<a A(c)$ and $a<\Lambda . \forall b<\mathbb{K} \exists ! x\left[x=\mathcal{H}_{a}(b)\right]$ follows from IH in ZFL. $\exists ! b \leq \kappa\left(b=\psi \psi_{\kappa}^{\vec{\xi}} a\right)$ follows from this.

2.82 Let $\pi$ be a weakly inaccessible cardinal with $f^{\prime \prime}(\{a\} \cup K(\xi)) \subset L_{\pi}$. Let $f$ be an injection such that $f^{\prime \prime} \mathcal{H}_{\Lambda}(\pi) \subset L_{\pi}$. Then for $\forall \alpha \in K(\xi)(f(\alpha) \in$ f" $\left.\mathcal{H}_{\alpha}(\pi)\right), \pi \in M h_{k}^{a}(\xi)$ iff for any $f(\vec{\nu})=\left(f\left(\nu_{k}\right), \ldots, f\left(\nu_{N-1}\right)\right)$, each of $f\left(\nu_{i}\right) \in$ $L_{\pi}$, if $\forall \alpha \in K(\vec{\nu})\left(f(\alpha) \in f^{\prime \prime} \mathcal{H}_{a}(\pi)\right)$ and $\vec{\nu}<\xi$, then $\pi \in M_{k}\left(M h_{k}^{a}(\vec{\nu})\right)$, where $f^{\prime \prime} \mathcal{H}_{a}(\pi) \subset L_{\pi}$ is a class in $L_{\pi}$.

2.83 We show the following $B(a)$ is progressive in $a<\Lambda$ :

$$
B(a): \Leftrightarrow \mathbb{K} \in M h_{N-1}^{\alpha}(a) \cap M_{N-1}\left(M h_{N-1}^{\alpha}(a)\right)
$$

Note that $a \in \mathcal{H}_{a}(\mathbb{K})$ holds for any $a<\Lambda$.

Suppose $\forall b<a B(b)$. We have to show that $M h_{N-1}^{\alpha}(a)$ is $\Pi_{N-3}^{1}$-indescribable in $\mathbb{K}$. It is easy to see that if $\pi \in M_{N-1}\left(M h_{N-1}^{\alpha}(a)\right)$, then $\pi \in M h_{N-1}^{\alpha}(a)$ by induction on $\pi$. Let $\theta(u)$ be a $\Pi_{N-3}^{1}$-formula such that $L_{\mathbb{K}} \models \theta(u)$.

By IH we have $\forall b<a\left[\mathbb{K} \in M_{N-1}\left(M h_{N-1}^{\alpha}(b)\right)\right]$. In other words, $\mathbb{K} \in$ $M h_{N-1}^{\alpha}(a)$, i.e., $L_{\mathbb{K}} \models m h_{N-1}^{\alpha}(a)$, where $m h_{N-1}^{\alpha}(a)$ is a $\Pi_{N-2}^{1}$-sentence in Proposition 2.822 Since the universe $L_{\mathbb{K}}$ is $\Pi_{N-2}^{1}$-indescribable, pick a $\pi<\mathbb{K}$ such that $L_{\pi}$ enjoys the $\Pi_{N-2}^{1}$-sentence $\theta(u) \wedge m h_{N-1}^{\alpha}(a)$, and $\{f(\alpha), f(a)\} \subset$ $L_{\pi}$. Therefore $\pi \in M h_{N-1}^{\alpha}(a)$ and $L_{\pi} \models \theta(u)$. Thus $\mathbb{K} \in M_{N-1}\left(M h_{N-1}^{\alpha}(a)\right)$.

\subsection{Normal forms in ordinal notations}

In this subsection we introduce an irreducibility of sequences, which is needed to define a normal form in ordinal notations.

Proposition $2.9 \pi \in M h_{k}^{a}(\zeta) \& \xi \leq \zeta \Rightarrow \pi \in M h_{k}^{a}(\xi)$.

Proof. (2) for $\pi \in M h_{k}^{a}(\xi)$ in Definition 2.512 follows from $\pi \in M h_{k}^{a}(\zeta)$ and Proposition 2.3 .

Lemma 2.10 (Cf. Lemma 3 in [1.) Assume $\mathbb{K} \geq \pi \in M h_{k}^{a}(\xi) \cap M h_{k+1}^{a}\left(\xi_{0}\right)$ with $2 \leq k \leq N-1$, he $(\mu) \leq \xi_{0}$ and $\{a\} \cup K(\mu) \subset \mathcal{H}_{a}(\pi)$. Then $\pi \in M h_{k}^{a}(\xi+\mu)$ holds. Moreover if $\pi \in M_{k+1}$, then $\pi \in M_{k+1}\left(M h_{k}^{a}(\xi+\mu)\right)$ holds. 
Proof. Suppose $\pi \in M h_{k}^{a}(\xi) \cap M h_{k+1}^{a}\left(\xi_{0}\right)$ and $K(\mu) \subset \mathcal{H}_{a}(\pi)$ with $h e(\mu) \leq$ $\xi_{0}$. We show $\pi \in M h_{k}^{a}(\xi+\mu)$ by induction on ordinals $\mu$. First note that if $b \in \mathcal{H}_{a}(\pi)$, then $f(b) \in f^{\prime \prime} \mathcal{H}_{\Lambda}(\pi) \subset L_{\pi}$. We have $K(\xi+\mu) \subset \mathcal{H}_{a}(\pi)$. $\pi \in M_{k+1}\left(M h_{k}^{a}(\xi+\mu)\right)$ follows from $\pi \in M h_{k}^{a}(\xi+\mu)$ and $\pi \in M_{k+1}$.

Let $(\zeta) * \vec{\nu}<\xi+\mu$ and $K(\zeta) \cup K(\vec{\nu}) \subset \mathcal{H}_{a}(\pi)$ for $\vec{\nu}=\left(\nu_{0}, \ldots, \nu_{n-1}\right)$. We need to show that $\pi \in M_{k}\left(M h_{k}^{a}((\zeta) * \vec{\nu})\right)$. By Definition 2.16, let $\left(\zeta_{0}\right) *\left(\mu_{0}, \ldots, \mu_{n-1}\right)$ be a sequence such that $\zeta<\zeta_{0} \leq_{p t} \xi+\mu, \mu_{0} \leq_{p t} t e\left(\zeta_{0}\right), \forall i \leq n-1\left(\nu_{i}<\mu_{i}\right)$, and $\forall i<n-1\left(\mu_{i+1} \leq \leq_{p t}\right.$ te $\left.\left(\mu_{i}\right)\right)$.

If $\zeta_{0} \leq_{p t} \xi$, then $(\zeta) * \vec{\nu}<\xi$, and $\pi \in M_{k}\left(M h_{k}^{a}((\zeta) * \vec{\nu})\right)$ by $\pi \in M h_{k}^{a}(\xi)$.

Let $\zeta_{0}=\xi+\zeta_{1}$ with $0<\zeta_{1} \leq_{p t} \mu$. If $\zeta_{1}<_{p t} \mu$, then by IH with he( $\left.\zeta_{1}\right)=$ $h e(\mu)$ we have $\pi \in M h_{k}^{a}\left(\zeta_{0}\right)$. On the other hand we have $(\zeta) * \vec{\nu}<\zeta_{0}$. Hence $\pi \in M_{k}\left(M h_{k}^{a}((\zeta) * \vec{\nu})\right)$.

Finally consider the case when $0<\zeta_{1}=\mu$. Then we obtain $\vec{\nu}<t e(\xi+$ $\mu)=t e(\mu) \leq h e(\mu) \leq \xi_{0} . \quad \pi \in M h_{k+1}^{a}\left(\xi_{0}\right)$ with Proposition 2.9 yields $\pi \in$ $M_{k+1}\left(M h_{k+1}^{a}(\vec{\nu})\right)$.

On the other side we see $\pi \in M h_{k}^{a}(\zeta)$ as follows. We have $\zeta<\xi+\mu$. If $\zeta \leq \xi$, then this follows from $\pi \in M h_{k}^{a}(\xi)$ and Proposition[2.9, and if $\zeta=\xi+\lambda<\xi+\mu$, then IH yields $\pi \in M h_{k}^{a}(\zeta)$.

Since $\pi \in M h_{k}^{a}(\zeta)$ is a $\Pi_{k-1}^{1}$-sentence holding on $L_{\pi}$ by Lemma 2.812 and $\{a\} \cup K(\zeta) \subset \mathcal{H}_{a}(\pi)$, we obtain $\pi \in M_{k+1}\left(M h_{k}^{a}((\zeta) * \vec{\nu})\right)$, a fortiori $\pi \in$ $M_{k}\left(M h_{k}^{a}((\zeta) * \vec{\nu})\right)$.

Definition 2.11 For sequences of ordinals $\vec{\xi}=\left(\xi_{k}, \ldots, \xi_{N-1}\right)$ and $\vec{\nu}=\left(\nu_{k}, \ldots, \nu_{N-1}\right)$ and $2 \leq k, m, n \leq N-1$,

$M h_{m}^{a}(\vec{\nu}) \prec_{k} M h_{n}^{a}(\vec{\xi}): \Leftrightarrow \forall \pi \in M h_{n}^{a}(\vec{\xi})\left(\{a, \pi\} \cup K(\vec{\nu}) \subset \mathcal{H}_{a}(\pi) \Rightarrow \pi \in M_{k}\left(M h_{m}^{a}(\vec{\nu})\right)\right)$.

Corollary 2.12 Let $\vec{\nu}$ be a sequence defined from a sequence $\vec{\xi}$ as follows. $\forall i<$ $k\left(\nu_{i}=\xi_{i}\right), \forall i>k\left(\nu_{i}=0\right)$, and $\nu_{k}=\xi_{k}+\Lambda^{\xi_{k+1}} b$, where $2 \leq k<N, b<\Lambda$ and $\xi_{k+1} \neq 0$. Then $M h_{2}^{a}(\vec{\nu}) \prec_{k+1} M h_{2}^{a}(\vec{\xi})$ holds. In particular if $\pi \in M h_{2}^{a}(\vec{\xi})$ and $K(\vec{\nu}) \cup\{\pi, a\} \subset \mathcal{H}_{a}(\pi)$, then $\psi_{\pi}^{\vec{\nu}}(a)<\pi$.

Proof. This is seen from Lemma 2.10.

Proposition 2.13 Let $\vec{\nu}=\left(\nu_{2}, \ldots, \nu_{N-1}\right), \vec{\xi}=\left(\xi_{2}, \ldots, \xi_{N-1}\right)$ be sequences of ordinals $<\varepsilon(\Lambda)$ such that $\vec{\nu}<_{k} \vec{\xi}$ for an integer $k$ with $2 \leq k \leq N-1$. Then $M h_{2}^{a}(\vec{\nu}) \prec_{k} M h_{2}^{a}(\vec{\xi})$. In particular if $\pi \in M h_{2}^{a}(\vec{\xi})$ and $K(\vec{\nu}) \cup\{\pi, a\} \subset \mathcal{H}_{a}(\pi)$, then $\psi_{\pi}^{\vec{\nu}}(a)<\pi$.

Proof. Assume $\pi \in M h_{2}^{a}(\vec{\xi})$ and $K(\vec{\nu}) \subset \mathcal{H}_{a}(\pi)$. We have $\pi \in M h_{k}^{a}\left(\xi_{k}\right)$. By the definition (2) and $\left(\nu_{k}, \ldots, \nu_{N-1}\right)<\xi_{k}$, we obtain $\pi \in M_{k}\left(\bigcap_{k \leq i<N-1} M h_{i}^{a}\left(\nu_{i}\right)\right)$.

On the other hand we have $\pi \in \bigcap_{i<k} M h_{i}^{a}\left(\xi_{i}\right)$, and hence $\pi \in \bigcap_{i<k} M h_{i}^{a}\left(\nu_{i}\right)$ by $\forall i<k\left(\nu_{i} \leq \xi_{i}\right)$ and Proposition [2.9. Since $\pi \in \bigcap_{i<k} M h_{i}^{a}\left(\nu_{i}\right)$ is a $\Pi_{k-2^{-}}^{1}$ sentence holding in $L_{\pi}$, we obtain $\pi \in M_{k}\left(\bigcap_{i \leq N-1} M h_{i}^{a}\left(\nu_{i}\right)\right)=M_{k}\left(M h_{2}^{a}(\vec{\nu})\right)$, a fortiori $\pi \in M_{2}\left(M h_{2}^{a}(\vec{\nu})\right)$.

Suppose $\{\pi, a\} \subset \mathcal{H}_{a}(\pi)$. The set $C=\left\{\kappa<\pi: \mathcal{H}_{a}(\kappa) \cap \pi \subset \kappa, K(\vec{\nu}) \cup\right.$ $\left.\{\pi, a\} \subset \mathcal{H}_{a}(\kappa)\right\}$ is a club subset of the regular cardinal $\pi$. This shows the 
existence of a $\kappa \in M h_{2}^{a}(\vec{\nu}) \cap C \cap \pi$, and hence $\psi_{\pi}^{\vec{\nu}}(a)<\pi$ by the definition (3).

Proposition 2.14 Let $\vec{\xi}=\left(\xi_{2}, \ldots, \xi_{N-1}\right)$ be a sequence of ordinals $<\varepsilon(\Lambda)$ such that $\{\pi, a\} \cup K(\vec{\xi}) \subset \mathcal{H}_{a}(\pi)$. Assume $\operatorname{Tl}\left(\xi_{i}\right)<\Lambda_{k}\left(\xi_{i+k}+1\right)$ for some $i<N-1$ and $k>0$. Then $\pi \in M h_{2}^{a}(\vec{\xi}) \Leftrightarrow \pi \in M h_{2}^{a}(\vec{\mu})$, where $\vec{\mu}=\left(\mu_{2}, \ldots, \mu_{N-1}\right)$ with $\mu_{i}=\xi_{i}-T l\left(\xi_{i}\right)$ and $\mu_{j}=\xi_{j}$ for $j \neq i$.

Proof. When $0<\xi_{i}=\Lambda^{\gamma_{m}} a_{m}+\cdots+\Lambda^{\gamma_{1}} a_{1}+\Lambda^{\gamma_{0}} a_{0}$ with $\gamma_{m}>\cdots>\gamma_{1}>$ $\gamma_{0}, 0<a_{i}<\Lambda, \mu_{i}=\Lambda^{\gamma_{m}} a_{m}+\cdots+\Lambda^{\gamma_{1}} a_{1}$ for $\operatorname{Tl}\left(\xi_{i}\right)=\Lambda^{\gamma_{0}} a_{0}$. If $\xi_{i}=0$, then so is $\mu_{i}=0$.

Let $\pi \in M h_{2}^{a}(\vec{\mu})$ and $T l\left(\xi_{i}\right)<\Lambda_{k}\left(\xi_{i+k}+1\right)$. We obtain $\forall j \leq k\left(h e^{(j)}\left(T l\left(\xi_{i}\right)\right)<\right.$ $\left.\Lambda_{k-j}\left(\xi_{i+k}+1\right)\right)$, and $h e^{(k)}\left(T l\left(\xi_{i}\right)\right) \leq \xi_{i+k}$. On the other hand we have $\pi \in$ $M h_{i+k}^{a}\left(\xi_{i+k}\right)$. From Lemma 2.10 we see inductively that for any $j<k, \pi \in$ $M h_{i+j}^{a}\left(h e^{(j)}\left(T l\left(\xi_{i}\right)\right)\right)$. In particular $\pi \in M h_{i+1}^{a}\left(h e\left(T l\left(\xi_{i}\right)\right)\right)$, and once again by Lemma 2.10 and $\pi \in M h_{i}^{a}\left(\mu_{i}\right)$ we obtain $\pi \in M h_{i}^{a}\left(\xi_{i}\right)$. Hence $\pi \in M h_{2}^{a}(\vec{\xi})$.

Definition 2.15 A sequence of ordinals $\vec{\xi}=\left(\xi_{2}, \ldots, \xi_{N-1}\right)$ is said to be irreducible iff $\forall i<N-1 \forall k>0\left(\xi_{i}>0 \Rightarrow T l\left(\xi_{i}\right) \geq \Lambda_{k}\left(\xi_{i+k}+1\right)\right)$.

Proposition 2.16 Let $\vec{\nu}=\left(\nu_{k}, \ldots, \nu_{N-1}\right) \neq \overrightarrow{0}$ be an irreducible sequence, and $k_{0} \geq k$ be the least number such that $\nu_{k_{0}} \neq 0$. Assume $\nu_{k_{0}}<h e^{\left(k_{0}-k\right)}(\xi)$. Then $\vec{\nu}<\xi$ holds in the sense of Definition 2.116.

Proof. Let $\ell<N-k$ be the largest number such that $\nu_{k+\ell} \neq 0$. We show $\left(\nu_{k}, \ldots, \nu_{k+\ell}\right)<\xi$. Since $\vec{\nu}$ is irreducible, we have $\Lambda_{i}\left(\nu_{k_{0}+i}+1\right) \leq \operatorname{Tl}\left(\nu_{k_{0}}\right)$. From $\nu_{k_{0}}<h e^{\left(k_{0}-k\right)}(\xi)$ and $t e(\mu) \leq h e(\mu)$ we obtain $\nu_{k_{0}+i}<\nu_{k_{0}+i}+1 \leq$ $h e^{(i)}\left(\nu_{k_{0}}\right) \leq h e^{\left(k_{0}-k+i\right)}(\xi)$. Let $\left(\mu_{k}, \ldots, \mu_{N-1}\right) \subset_{p t} \xi$ such that $\mu_{k}=H d(\xi)$ and $\mu_{i+1}=h e\left(\mu_{i}\right)=t e\left(H d\left(\mu_{i}\right)\right)$. Then $t e\left(\mu_{k+i}\right)=h e\left(\mu_{k+i}\right)$ and $\mu_{k_{0}+i}=$ $h e\left(\mu_{k_{0}+i-1}\right)=h e^{\left(k_{0}-k+i\right)}(\xi)$ for $k_{0}-k+i>0$. Therefore $\left(\mu_{k}, \ldots, \mu_{k+\ell}\right) \subset p t \xi$ witnesses $\left(\nu_{k}, \ldots, \nu_{k+\ell}\right)<\xi$.

Definition 2.17 Let $\vec{\xi}=\left(\xi_{k}, \ldots, \xi_{N-1}\right), \vec{\nu}=\left(\nu_{k}, \ldots, \nu_{N-1}\right)$ and $\vec{\nu} \neq \vec{\xi}$. Let $i \geq k$ be the minimal number such that $\nu_{i} \neq \xi_{i}$. Suppose $\left(\xi_{i}, \ldots, \xi_{N-1}\right) \neq \overrightarrow{0}$, and let $k_{1} \geq i$ be the minimal number such that $\xi_{k_{1}} \neq 0$. Then $\vec{\nu}<_{l x, k} \vec{\xi}$ iff one of the followings holds:

1. $\left(\nu_{i}, \ldots, \nu_{N-1}\right)=\overrightarrow{0}$.

2. In what follows assume $\left(\nu_{i}, \ldots, \nu_{N-1}\right) \neq \overrightarrow{0}$, and let $k_{0} \geq i$ be the minimal number such that $\nu_{k_{0}} \neq 0\left(i=\min \left\{k_{0}, k_{1}\right\}\right)$. Then $\vec{\nu}<_{l x, k} \vec{\xi}$ iff one of the followings holds:

(a) $i=k_{0}<k_{1}$ and $h e^{\left(k_{1}-k_{0}\right)}\left(\nu_{k_{0}}\right) \leq \xi_{k_{1}}$.

(b) $k_{0} \geq k_{1}=i$ and $\nu_{k_{0}}<h e^{\left(k_{0}-k_{1}\right)}\left(\xi_{k_{1}}\right)$.

Proposition 2.18 Suppose that both of $\vec{\nu}$ and $\vec{\xi}$ are irreducible. Then $\vec{\nu}<_{l x, k}$ $\vec{\xi} \Rightarrow M h_{k}^{a}(\vec{\nu}) \prec_{k} M h_{k}^{a}(\vec{\xi})$. 
Proof. Let $\pi \in M h_{k}^{a}(\vec{\xi}), K(\vec{\nu}) \subset \mathcal{H}_{a}(\pi)$, and $i \geq k$ be the minimal number such that $\nu_{i} \neq \xi_{i}$. We have $\pi \in \bigcap_{k \leq j<i} M h_{j}^{a}\left(\nu_{j}\right)$, which is a $\Pi_{i-2}^{1}$-sentence holding on $L_{\pi}$. In the case $\xi_{i} \neq 0$, it suffices to show that $\pi \in M_{i}\left(\bigcap_{j \geq i} M h_{j}^{a}\left(\nu_{j}\right)\right)$, since then we obtain $\pi \in M_{i}\left(M h_{k}^{a}(\vec{\nu})\right)$ by $\pi \in M h_{i}^{a}\left(\xi_{i}\right) \subset M_{i}$, a fortiori $\pi \in M_{k}\left(M h_{k}^{a}(\vec{\nu})\right)$.

If $\left(\nu_{i}, \ldots, \nu_{N-1}\right)=\overrightarrow{0}$, then $\xi_{i} \neq 0$ and $\bigcap_{j>i} M h_{j}^{a}\left(\nu_{j}\right)$ denotes the class of limit ordinals. Obviously $\pi \in M_{i}\left(\bigcap_{j \geq i} M h_{j}^{a}\left(\nu_{j}\right)\right)$.

In what follows assume $\left(\nu_{i}, \ldots, \nu_{N-1}\right) \neq \overrightarrow{0}$, and let $k_{0} \geq i$ be the minimal number such that $\nu_{k_{0}} \neq 0$, and $k_{1} \geq i$ be the minimal number such that $\xi_{k_{1}} \neq 0$. Case 1. $k_{0} \geq k_{1}=i$ : Then we have $\nu_{k_{0}}<h e^{\left(k_{0}-k_{1}\right)}\left(\xi_{k_{1}}\right)$. Proposition 2.16 yields $\left(\nu_{k_{0}}, \ldots, \nu_{N-1}\right)<\xi_{k_{1}}=\xi_{i}$, which in turn yields $\pi \in M_{i}\left(\bigcap_{j \geq i} M h_{j}^{a}\left(\nu_{j}\right)\right)$ by the definition (2) of $\pi \in M h_{i}^{a}\left(\xi_{i}\right)$.

Case 2. $i=k_{0}<k_{1}$ : Then we have $h e^{\left(k_{1}-i\right)}\left(\nu_{i}\right) \leq \xi_{k_{1}}$. Also $\nu_{i+p}<h e^{(p)}\left(\nu_{i}\right)$ for any $p>0$ since $\vec{\nu}$ is irreducible and $\nu_{i} \neq 0$. Let $j \geq k_{1}$. Then $\nu_{j}<h e^{(j-i)}\left(\nu_{i}\right) \leq$ $h e^{\left(j-k_{1}\right)}\left(\xi_{k_{1}}\right)$. In particular $\nu_{k_{1}}<\xi_{k_{1}}$. Proposition 2.16yields $\left(\nu_{k_{1}}, \ldots, \nu_{N-1}\right)<$ $\xi_{k_{1}} . \quad \pi \in M h_{k_{1}}^{a}\left(\xi_{k_{1}}\right)$ yields $\pi \in M_{k_{1}}\left(\bigcap_{j \geq k_{1}} M h_{j}^{a}\left(\nu_{j}\right)\right)$. Moreover for any $p<$ $k_{1}-i, h e^{\left(k_{1}-i-p\right)}\left(\nu_{i+p}\right) \leq \xi_{k_{1}}$ by Proposition 2.2. Lemma 2.10 yields $\pi \in$ $\bigcap_{k_{1}>j \geq i} M h_{j}^{a}\left(\nu_{j}\right)$. Therefore $\pi \in M_{k_{1}}\left(M h_{k}^{a}(\vec{\nu})\right)$, a fortiori $\pi \in M_{k}\left(M h_{k}^{a}(\vec{\nu})\right)$.

Proposition 2.19 (Cf. Proposition 4.20 in [ 8 )

Let $\vec{\nu}=\left(\nu_{2}, \ldots, \nu_{N-1}\right), \vec{\xi}=\left(\xi_{2}, \ldots, \xi_{N-1}\right)$ be irreducible sequences of ordinals $<$ $\varepsilon(\Lambda)$, and assume that $\psi_{\pi}^{\vec{\nu}}(b)<\pi$ and $\psi_{\kappa}^{\vec{\xi}}(a)<\kappa$.

Then $\beta_{1}=\psi_{\pi}^{\vec{\nu}}(b)<\psi_{\kappa}^{\vec{\xi}}(a)=\alpha_{1}$ iff one of the following cases holds:

1. $\pi \leq \psi_{\kappa}^{\vec{\xi}}(a)$.

2. $b<a, \psi_{\pi}^{\vec{\nu}}(b)<\kappa$ and $K(\vec{\nu}) \cup\{\pi, b\} \subset \mathcal{H}_{a}\left(\psi_{\kappa}^{\vec{\xi}}(a)\right)$.

3. $b>a$ and $K(\vec{\xi}) \cup\{\kappa, a\} \not \subset \mathcal{H}_{b}\left(\psi_{\pi}^{\vec{\nu}}(b)\right)$.

4. $b=a, \kappa<\pi$ and $\kappa \notin \mathcal{H}_{b}\left(\psi_{\pi}^{\vec{v}}(b)\right)$.

5. $b=a, \pi=\kappa, K(\vec{\nu}) \subset \mathcal{H}_{a}\left(\psi_{\kappa}^{\vec{\xi}}(a)\right)$, and $\vec{\nu}<_{l x, 2} \vec{\xi}$.

6. $b=a, \pi=\kappa, K(\vec{\xi}) \not \subset \mathcal{H}_{b}\left(\psi_{\pi}^{\vec{\nu}}(b)\right)$.

Proof. If the case (2) holds, then $\psi_{\pi}^{\vec{\nu}}(b) \in \mathcal{H}_{a}\left(\psi_{\kappa}^{\vec{\xi}}(a)\right) \cap \kappa \subset \psi_{\kappa}^{\vec{\xi}}(a)$.

If one of the cases (3) and (41) holds, then $K(\vec{\xi}) \cup\{\kappa, a\} \not \subset \mathcal{H}_{a}\left(\psi_{\pi}^{\vec{\nu}}(b)\right)$. On the other hand we have $K(\vec{\xi}) \cup\{\kappa, a\} \subset \mathcal{H}_{a}\left(\psi_{\kappa}^{\vec{\xi}}(a)\right)$. Hence $\psi_{\pi}^{\vec{\nu}}(b)<\psi_{\kappa}^{\vec{\xi}}(a)$.

If the case (5D) holds, then Proposition 2.18 yields $M h_{2}^{a}(\vec{\nu}) \prec_{2} M h_{2}^{a}(\vec{\xi}) \ni$ $\psi_{\kappa}^{\vec{\xi}}(a)$. Hence $\psi_{\kappa}^{\vec{\xi}}(a) \in M_{2}\left(M h_{2}^{a}(\vec{\nu})\right)$. Since $K(\vec{\nu}) \cup\{\kappa, a\} \subset \mathcal{H}_{a}\left(\psi_{\kappa}^{\vec{\xi}}(a)\right)$, the set $\left\{\rho<\psi_{\kappa}^{\vec{\xi}}(a): \mathcal{H}_{a}(\rho) \cap \kappa \subset \rho, K(\vec{\nu}) \cup\{\kappa, a\} \subset \mathcal{H}_{a}(\rho)\right\}$ is club in $\psi_{\kappa}^{\vec{\xi}}(a)$. Therefore $\psi_{\pi}^{\vec{\nu}}(b)=\psi_{\kappa}^{\vec{\nu}}(a)<\psi_{\kappa}^{\vec{\xi}}(a)$ by (3) in Definition 2.513.

Finally assume that the case (16) holds. Since $K(\vec{\xi}) \subset \mathcal{H}_{a}\left(\psi_{\kappa}^{\vec{\xi}}(a)\right), \psi_{\pi}^{\vec{\nu}}(b)<$ $\psi_{\kappa}^{\vec{\xi}}(a)$ holds. 
Conversely assume that $\psi_{\pi}^{\vec{\nu}}(b)<\psi_{\kappa}^{\vec{\xi}}(a)$ and $\psi_{\kappa}^{\vec{\xi}}(a)<\pi$.

First consider the case $b<a$. Then we have $K(\vec{\nu}) \cup\{\pi, b\} \subset \mathcal{H}_{b}\left(\psi_{\pi}^{\vec{\nu}}(b)\right) \subset$ $\mathcal{H}_{a}\left(\psi_{\kappa}^{\vec{\xi}}(a)\right)$. Hence (2) holds.

Next consider the case $b>a . K(\vec{\xi}) \cup\{\kappa, a\} \subset \mathcal{H}_{b}\left(\psi_{\pi}^{\vec{\nu}}(b)\right)$ would yield $\psi_{\kappa}^{\vec{\xi}}(a) \in$ $\mathcal{H}_{b}\left(\psi_{\pi}^{\vec{v}}(b)\right) \cap \pi \subset \psi_{\pi}^{\vec{\nu}}(b)$, a contradiction $\psi_{\kappa}^{\vec{\xi}}(a)<\psi_{\pi}^{\vec{\nu}}(b)$. Hence (3) holds.

Finally assume $b=a$. Consider the case $\kappa<\pi . \kappa \in \mathcal{H}_{b}\left(\psi_{\pi}^{\vec{\nu}}(b)\right) \cap \pi$ would yield $\psi_{\kappa}^{\vec{\xi}}(a)<\kappa<\psi_{\pi}^{\vec{\nu}}(b)$, a contradiction. Hence $\kappa \notin \mathcal{H}_{b}\left(\psi_{\pi}^{\vec{\nu}}(b)\right)$, and (4) holds. If $\pi<\kappa$, then $\pi \in \mathcal{H}_{b}\left(\psi_{\pi}^{\vec{v}}(b)\right) \cap \kappa \subset \mathcal{H}_{a}\left(\psi_{\kappa}^{\vec{\xi}}(a)\right) \cap \kappa$, and $\pi<\psi_{\kappa}^{\vec{\xi}}(a)$, a contradiction, or we should say that (11) holds. Finally let $\pi=\kappa$. We can assume that $K(\vec{\xi}) \subset \mathcal{H}_{b}\left(\psi_{\pi}^{\vec{\nu}}(b)\right)$, otherwise (6) holds. If $\vec{\xi}<_{l x, 2} \vec{\nu}$, then by (5) $\psi_{\kappa}^{\vec{\xi}}(a)<\psi_{\pi}^{\vec{\nu}}(b)$ would follow. If $K(\vec{\nu}) \not \subset \mathcal{H}_{a}\left(\psi_{\kappa}^{\vec{\xi}}(a)\right)$, then by (6) again $\psi_{\kappa}^{\vec{\xi}}(a)<\psi_{\pi}^{\vec{\nu}}(b)$ would follow. Hence $K(\vec{\nu}) \subset \mathcal{H}_{a}\left(\psi_{\kappa}^{\vec{\xi}}(a)\right)$ and $\vec{\nu} \leq_{l x} \vec{\xi}$. If $\vec{\nu}=\vec{\xi}$, then $\psi_{\kappa}^{\vec{\xi}}(a)=\psi_{\pi}^{\vec{\nu}}(b)$. Therefore (5) must be the case.

Definition 2.20 is utilized to define a computable notation system in the next section 3 ,

Definition 2.20 A set $S D$ of sequences $\vec{\xi}=\left(\xi_{2}, \ldots, \xi_{N-1}\right)$ of ordinals $\xi_{i}<\varepsilon(\Lambda)$ is defined recursively as follows.

1. $\overrightarrow{0} *(a) \in S D$ for each $a<\Lambda$.

2. (Cf. Definition 2.19) Let $\vec{\xi}=\left(\xi_{2}, \ldots, \xi_{N-1}\right) \in S D, 1 \leq k<N-1, \zeta<$ $\varepsilon(\Lambda)$ be an ordinal such that $\left(\xi_{k+1}, \ldots, \xi_{N-1}\right)<_{s d} \zeta$, and $\left(\xi_{2}, \ldots, \xi_{k-1}, \xi_{k}, \zeta\right) *$ $\overrightarrow{0} \in S D$. Then for $\zeta_{k}=\xi_{k}+\Lambda^{\zeta} a$ with an ordinal $a<\Lambda,\left(\xi_{2}, \ldots, \xi_{k-1}\right) *$ $\left(\zeta_{k}\right) *\left(\xi_{k+1}, \ldots, \xi_{N-1}\right) \in S D$ and $\left(\xi_{2}, \ldots, \xi_{k-1}\right) *\left(\zeta_{k}\right) * \overrightarrow{0} \in S D$.

Proposition 2.21 Let $\vec{\xi}=\left(\xi_{2}, \ldots, \xi_{N-1}\right) \in S D$.

1. $\left(\xi_{2}, \ldots, \xi_{i}\right) * \overrightarrow{0} \in S D$ for each $i$ with $1 \leq i<N$.

2. For $2 \leq i<j<k<N$, if $\xi_{i} \neq 0$ and $\xi_{k} \neq 0$, then $\xi_{j} \neq 0$.

3. Let $\xi_{i} \neq 0$. Then $\left(\xi_{i+1}, \ldots, \xi_{N-1}\right)<_{s d}$ te $\left(\xi_{i}\right)$.

4. $\vec{\xi}$ is irreducible.

Proof. Let $1 \leq k<N-1, \zeta<\varepsilon(\Lambda)$ be an ordinal such that $\left(\xi_{k+1}, \ldots, \xi_{N-1}\right)<_{s d}$ $\zeta$, and $\left(\xi_{2}, \ldots, \xi_{k-1}, \xi_{k}, \zeta\right) * \overrightarrow{0} \in S D$. Also let $\zeta_{k}=\xi_{k}+\Lambda^{\zeta} a$ with an ordinal $a<\Lambda$.

2.211 is seen by induction on the recursive definition of $\vec{\xi} \in S D$.

2.212 is seen by induction on the recursive definition of $\vec{\xi} \in S D$. Suppose $\xi_{i} \neq 0$ for an $i<k$. From $\left(\xi_{2}, \ldots, \xi_{k-1}, \xi_{k}, \zeta\right) * \overrightarrow{0} \in S D$ and $\zeta \neq 0$, IH yields $\xi_{k} \neq 0$.

2.2113 and 2.2114. We show these by simultaneous induction on the recursive definition of $\vec{\xi} \in S D$.

2.2113. We show Proposition 2.2113 for the sequence $\left(\xi_{2}, \ldots, \xi_{k-1}\right) *\left(\zeta_{k}\right) *$ $\left(\xi_{k+1}, \ldots, \xi_{N-1}\right) \in S D$. The proposition holds for the sequence $\vec{\xi}$, and we can 
assume $a \neq 0$. We obtain $\left(\xi_{i+1}, \ldots, \xi_{N-1}\right)<_{s d}$ te $\left(\xi_{i}\right)$ for $i>k$ if $\xi_{i} \neq 0$, and $\left(\xi_{k+1}, \ldots, \xi_{N-1}\right)<_{s d} t e\left(\zeta_{k}\right)=\zeta$ by the assumption. Let $2 \leq i<k$ and $\xi_{i} \neq 0$. We show $\left(\xi_{i+1}, \ldots, \xi_{k-1}\right) *\left(\zeta_{k}\right) *\left(\xi_{k+1}, \ldots, \xi_{N-1}\right)<_{s d} t e\left(\xi_{i}\right)$. It suffices to show that $\zeta_{k}<_{s d} t e^{(k-i)}\left(\xi_{i}\right)$. By IH we have $\xi_{k}<_{s d} t e^{(k-i)}\left(\xi_{i}\right)$. On the other hand we have $\xi_{k} \neq 0$ by $\left(\xi_{2}, \ldots, \xi_{k-1}, \xi_{k}, \zeta\right) * \overrightarrow{0} \in S D, \zeta \neq 0$, and Proposition 2.212 Moreover $\left(\xi_{2}, \ldots, \xi_{k-1}, \xi_{k}, \zeta\right) * \overrightarrow{0}$ is irreducible by Proposition 2.214 and hence $T l\left(\xi_{k}\right) \geq \Lambda^{\zeta+1}$. Therefore $t e\left(\xi_{k}\right)>\zeta$. This means that $\zeta_{k}={ }_{N F} \xi_{k}+\Lambda^{\zeta} a$, and $\xi_{k}<_{s d} t e^{(k-i)}\left(\xi_{i}\right)$ yields $\zeta_{k}<_{s d} t e^{(k-i)}\left(\xi_{i}\right)$ by Definition 2.118.

2.2114] If $\left(\xi_{i+1}, \ldots, \xi_{N-1}\right)<_{s d} t e\left(\xi_{i}\right)$ for $\xi_{i} \neq 0$, then $\xi_{i+k}<_{s d} t e^{(k)}\left(\xi_{i}\right)$ for $k>0$, and $\xi_{i+k}+1 \leq t e^{(k)}\left(\xi_{i}\right)$. Hence $\Lambda_{k}\left(\xi_{i+k}+1\right) \leq \Lambda^{t e\left(\xi_{i}\right)} \leq T l\left(\xi_{i}\right)$, and $\vec{\xi}$ is irreducible.

\section{Computable notation system $O T$}

In this section (except Propositions 3.3) we work in a weak fragment of arithmetic, e.g., in the fragment $I \Sigma_{1}$ or even in the bounded arithmetic $S_{2}^{1}$. Referring Proposition 2.19 the sets of ordinal terms $O T \subset \Lambda=\varepsilon_{\mathbb{K}+1}$ and $E \subset \varepsilon(\Lambda)=\varepsilon_{\mathbb{K}+2}$ over symbols $\{0, \mathbb{K}, \Lambda,+, \omega, \varphi, \Omega, \psi\}$ are defined recursively. $O T$ is isomorphic to a subset of $\mathcal{H}_{\Lambda}(0)$. Simultaneously we define finite sets $K_{\delta}(\alpha) \subset O T$ for $\delta, \alpha \in O T$, and sequences $\left(m_{k}(\alpha)\right)_{2 \leq k \leq N-1}$ for $\alpha \in O T \cap \mathbb{K}$, where in $\alpha=\psi_{\pi}^{\vec{v}}(a)$, $m_{k}(\alpha)=\nu_{k}$, i.e., $\vec{\nu}=\left(\nu_{2}, \ldots, \nu_{N-1}\right)=\left(m_{2}(\alpha), \ldots, m_{N-1}(\alpha)\right)=\left(m_{k}(\alpha)\right)_{k}=$ $\vec{m}(\alpha)$. For $\left\{\alpha_{0}, \ldots, \alpha_{m}, \beta\right\} \subset O T, K_{\delta}\left(\alpha_{0}, \ldots, \alpha_{m}\right):=\bigcup_{i \leq m} K_{\delta}\left(\alpha_{i}\right), K_{\delta}\left(\alpha_{0}, \ldots, \alpha_{m}\right)<$ $\beta: \Leftrightarrow \forall \gamma \in K_{\delta}\left(\alpha_{0}, \ldots, \alpha_{m}\right)(\gamma<\beta)$, and $\beta \leq K_{\delta}\left(\alpha_{0}, \ldots, \alpha_{m}\right): \Leftrightarrow \exists \gamma \in K_{\delta}\left(\alpha_{0}, \ldots, \alpha_{m}\right)(\beta \leq$ $\gamma)$.

An ordinal term in $O T$ is said to be a regular term if it is one of the form $\mathbb{K}, \Omega_{\beta+1}$ or $\psi_{\tau}^{\vec{\nu}}(a)$ with the non-zero sequences $\vec{\nu} \neq \overrightarrow{0}$. $\mathbb{K}$ and the latter terms $\psi_{\pi}^{\vec{\nu}}(a)$ are Mahlo terms.

$\alpha={ }_{N F} \alpha_{m}+\cdots+\alpha_{0}$ means that $\alpha=\alpha_{m}+\cdots+\alpha_{0}$ and $\alpha_{m} \geq \cdots \geq \alpha_{0}$ and each $\alpha_{i}$ is a non-zero additive principal number. $\alpha=_{N F} \varphi \beta \gamma$ means that $\alpha=\varphi \beta \gamma$ and $\beta, \gamma<\alpha . \alpha={ }_{N F} \omega^{\beta}$ means that $\alpha=\omega^{\beta}>\beta . \alpha={ }_{N F} \Omega_{\beta}$ means that $\alpha=\Omega_{\beta}>\beta$.

Let $p d\left(\psi_{\pi}^{\vec{\nu}}(a)\right)=\pi$ (even if $\vec{\nu}=\overrightarrow{0}$ ). Moreover for $n, p d^{(n)}(\alpha)$ is defined recursively by $p d^{(0)}(\alpha)=\alpha$ and $p d^{(n+1)}(\alpha) \simeq p d\left(p d^{(n)}(\alpha)\right)$.

For terms $\pi, \kappa \in O T, \pi \prec \kappa$ denotes the transitive closure of the relation $\left\{(\pi, \kappa): \exists \vec{\xi} \exists b\left[\pi=\psi_{\kappa}^{\vec{\xi}}(b)\right]\right\}$, and its reflexive closure $\pi \preceq \kappa: \Leftrightarrow \pi \prec \kappa \vee \pi=\kappa \Leftrightarrow$ $\exists n\left(\kappa=p d^{(n)}(\pi)\right)$.

For each ordinal term $\alpha=\psi_{\pi}^{\vec{\nu}}(a)$, a series $\left(\pi_{i}\right)_{i \leq L}$ of ordinal terms is uniquely determined as follows: $\pi_{L}=\alpha, \pi_{i}=p d\left(\pi_{i+1}\right)$ and $\pi_{0}=\mathbb{K}$. Let us call the series $\left(\pi_{i}\right)_{i \leq L}$ the collapsing series of $\alpha=\pi_{L}$.

Then we see that an ordinal term $\alpha=\psi_{\pi}^{\vec{\nu}}(a)$ with $\vec{\nu} \neq \overrightarrow{0}$ is constructed by Definition [3.12g below iff $L=1 . \alpha$ is constructed by Definition 3.121 iff $L \equiv 1$ $(\bmod (N-2))$. Otherwise $\alpha$ is constructed by Definition $3.12 \mathrm{~h}$

Definition $3.1 \ell \alpha$ denotes the number of occurrences of symbols $\{0, \mathbb{K}, \Lambda,+, \omega, \varphi, \Omega, \psi\}$ in terms $\alpha \in O T \cup E$. 
1. (a) $0 \in E$.

(b) If $0<a \in O T$, then $a \in E . K(a)=\{a\}$.

(c) If $\left\{\xi_{i}: i \leq m\right\} \subset E, \xi_{m}>\cdots>\xi_{0}>0$ and $0<b_{i} \in O T$, then $\sum_{i \leq m} \Lambda^{\xi_{i}} b_{i}=\Lambda^{\xi_{m}} b_{m}+\cdots+\Lambda^{\xi_{0}} b_{0} \in E . K\left(\sum_{i \leq m} \Lambda^{\xi_{i}} b_{i}\right)=\left\{b_{i}: i \leq\right.$ $m\} \cup \bigcup\left\{K\left(\xi_{i}\right): i \leq m\right\}$.

(d) For sequences $\vec{\nu}=\left(\nu_{2}, \ldots, \nu_{N-1}\right)$, let $K(\vec{\nu})=\bigcup_{2 \leq i \leq N-1} K\left(\nu_{i}\right)$.

2. (a) $0, \mathbb{K} \in O T$. $m_{k}(0)=0$ for any $k$, and $K_{\delta}(0)=K_{\delta}(\mathbb{K})=\emptyset$.

(b) If $\alpha={ }_{N F} \alpha_{m}+\cdots+\alpha_{0}(m>0)$ with $\left\{\alpha_{i}: i \leq m\right\} \subset O T$, then $\alpha \in O T$, and $m_{k}(\alpha)=0$ for any $k . K_{\delta}(\alpha)=K_{\delta}\left(\alpha_{0}, \ldots, \alpha_{m}\right)$.

(c) If $\alpha={ }_{N F} \varphi \beta \gamma$ with $\{\beta, \gamma\} \subset O T \cap \mathbb{K}$, then $\alpha \in O T$, and $m_{k}(\alpha)=0$ for any $k . K_{\delta}(\alpha)=K_{\delta}(\beta, \gamma)$.

(d) If $\alpha={ }_{N F} \omega^{\beta}$ with $\mathbb{K}<\beta \in O T$, then $\alpha \in O T$, and $m_{k}(\alpha)=0$ for any $k . K_{\delta}(\alpha)=K_{\delta}(\beta)$.

(e) If $\alpha={ }_{N F} \Omega_{\beta}$ with $\beta \in O T \cap \mathbb{K}$, then $\alpha \in O T \cdot m_{2}(\alpha)=1, m_{k}(\alpha)=0$ for any $k>2$ if $\beta$ is a successor ordinal. Otherwise $m_{k}(\alpha)=0$ for any $k$. In each case $K_{\delta}(\alpha)=K_{\delta}(\beta)$.

(f) Let $\alpha=\psi_{\pi}(a):=\psi_{\pi}^{\overrightarrow{0}}(a)$ where $\pi$ is a regular term, i.e., either $\pi=\mathbb{K}$ or $\vec{m}(\pi) \neq \overrightarrow{0}$, and $K_{\alpha}(\pi, a)<a$.

Then $\alpha=\psi_{\pi}(a) \in O T$. Let $m_{k}(\alpha)=0$ for any $k$. $K_{\delta}\left(\psi_{\pi}(a)\right)=\emptyset$ if $\alpha<\delta . K_{\delta}\left(\psi_{\pi}(a)\right)=\{a\} \cup K_{\delta}(a, \pi)$ otherwise.

(g) Let $\alpha=\psi_{\mathbb{K}}^{\vec{\nu}}(a)$ with $\vec{\nu}=\overrightarrow{0} *(b)(\operatorname{lh}(\vec{\nu})=N-2)$ and $b, a \in O T$ such that $0<b \leq a$ and $K_{\alpha}(b, a)<a$.

Then $\alpha=\psi_{\mathbb{K}}^{\vec{v}}(a) \in O T$. Let $m_{N-1}(\alpha)=b, m_{k}(\alpha)=0$ for $k<N-1$. $K_{\delta}\left(\psi_{\mathbb{K}}^{\vec{\nu}}(a)\right)=\emptyset$ if $\alpha<\delta . K_{\delta}\left(\psi_{\mathbb{K}}^{\vec{\nu}}(a)\right)=\{a\} \cup \bigcup\left\{K_{\delta}(\gamma): \gamma \in K(\nu)\right\}$ otherwise.

(h) Let $\pi \in O T \cap \mathbb{K}$ be such that $m_{k+1}(\pi) \neq 0$ and $\forall i>k+1\left(m_{i}(\pi)=0\right)$ for a $k(2 \leq k \leq N-2)$, and $b, a \in O T$ such that $0<b \leq a$. Let $\vec{\nu}=\left(\nu_{2}, \ldots, \nu_{N-1}\right)$ be a sequence defined by $\forall i<k\left(\nu_{i}=m_{i}(\pi)\right)$, $\nu_{k}=m_{k}(\pi)+\Lambda^{m_{k+1}(\pi)} b$, and $\forall i>k\left(\nu_{i}=0\right)$.

Then $\alpha=\psi_{\pi}^{\vec{\nu}}(a) \in O T$ if $K_{\alpha}(\pi, a, b) \cup K_{\alpha}(K(\vec{m}(\pi)))<a$. Let $m_{i}(\alpha)=\nu_{i}$ for each $i . K_{\delta}\left(\psi_{\pi}^{\vec{\nu}}(a)\right)=\emptyset$ if $\alpha<\delta$. Otherwise $K_{\delta}\left(\psi_{\pi}^{\vec{\nu}}(a)\right)=$ $\{a\} \cup K_{\delta}(a, \pi) \cup \bigcup\left\{K_{\delta}(b): b \in K(\vec{\nu})\right\}$.

(i) Let $\pi \in O T \cap \mathbb{K}$ be such that $m_{2}(\pi) \neq 0$ and $\forall i>2\left(m_{i}(\pi)=0\right)$, and $a \in O T$. Let $\overrightarrow{0} \neq \vec{\nu}=\left(\nu_{2}, \ldots, \nu_{N-1}\right) \in S D$ be a sequence of ordinal terms $\nu_{i} \in E$ such that $\vec{\nu}<_{s p} m_{2}(\pi)$.

Then $\alpha=\psi_{\pi}^{\vec{\nu}}(a)$ if $K_{\alpha}(\pi, a)<a$, and

$$
\forall k\left(K_{\alpha}\left(\nu_{k}\right)<\max K\left(\nu_{k}\right)\right)
$$

Let $m_{i}(\alpha)=\nu_{i}$ for each $i$.

$K_{\delta}\left(\psi_{\pi}^{\vec{\nu}}(a)\right)=\emptyset$ if $\alpha<\delta$. Otherwise $K_{\delta}\left(\psi_{\pi}^{\vec{\nu}}(a)\right)=\{a\} \cup K_{\delta}(a, \pi) \cup$ $\bigcup\left\{K_{\delta}(b): b \in K(\vec{\nu})\right\}$. 
Let $\{\pi, a, \xi\} \subset \mathcal{H}_{a}(\pi)$. Then $\xi=m_{k}(\pi)$ is intended to be equivalent to $\pi \in M h_{k}^{a}(\xi)$. For Definition 3.12h, see Corollary 2.12, and for Definition 3.12i see Proposition 2.13.

Proposition 3.2 For each Mahlo term $\alpha=\psi_{\pi}^{\vec{\nu}}(a) \in O T, \vec{m}(\alpha)=\vec{\nu} \in S D$ for the class $S D$ in Definition 2.20.

Proposition 3.3 For any $\alpha \in O T$ and any $\delta$ such that $\delta=0, \mathbb{K}$ or $\delta=\psi_{\pi}^{\vec{\nu}}(b)$ for some $\pi, b, \vec{\nu}, \alpha \in \mathcal{H}_{\gamma}(\delta) \Leftrightarrow K_{\delta}(\alpha)<\gamma$.

Proof. By induction on $\ell \alpha$.

Lemma $3.4(O T,<)$ is a computable notation system of ordinals. In particular the order type of the initial segment $\left\{\alpha \in O T: \alpha<\Omega_{1}\right\}$ is less than $\omega_{1}^{C K}$.

Specifically each of $\alpha<\beta$ and $\alpha=\beta$ is decidable for $\alpha, \beta \in O T$, and $\alpha \in O T$ is decidable for terms $\alpha$ over symbols $\{0, \mathbb{K}, \Lambda,+, \omega, \varphi, \Omega, \psi\}$.

Proof. These are shown simultaneously referring Propositions 2.19 and 3.3 Let us give recursive definitions only for terms $\Omega_{\alpha}, \psi_{\kappa}^{\vec{\nu}}(a) \in O T$.

First $\Omega_{\psi_{\kappa}^{\vec{\nu}}(a)}=\psi_{\kappa}^{\vec{\nu}}(a)$, i.e., $\Omega_{\alpha}<\psi_{\kappa}^{\vec{\nu}}(a) \Leftrightarrow \alpha<\psi_{\kappa}^{\vec{\nu}}(a), \psi_{\kappa}^{\vec{\nu}}(a)<\Omega_{\alpha} \Leftrightarrow$ $\psi_{\kappa}^{\vec{\nu}}(a)<\alpha$. Next $\Omega_{\alpha}<\psi_{\Omega_{\alpha+1}}(a)<\Omega_{\alpha+1}$.

Finally for $\psi_{\pi}^{\vec{\nu}}(b), \psi_{\kappa}^{\vec{\xi}}(a) \in O T, \psi_{\pi}^{\vec{\nu}}(b)<\psi_{\kappa}^{\vec{\xi}}(a)$ iff one of the following cases holds:

1. $\pi \leq \psi_{\kappa}^{\vec{\xi}}(a)$.

2. $b<a, \psi_{\pi}^{\vec{\nu}}(b)<\kappa$, and $K_{\psi_{\kappa}^{\vec{\xi}}(a)}(\{\pi, b\} \cup K(\vec{\nu}))<a$.

3. $b \geq a$, and $b \leq K_{\psi_{\pi}^{\vec{v}}(b)}(\{\kappa, a\} \cup K(\vec{\xi}))$.

4. $b=a, \pi=\kappa, K_{\psi_{\kappa}^{\vec{\xi}(a)}}(K(\vec{\nu}))<a$, and $\vec{\nu}<_{l x, 2} \vec{\xi}$.

Proposition 3.5 1. Let $\beta=\psi_{\pi}^{\vec{\nu}}(b)$ with $\pi=\psi_{\kappa}^{\vec{\xi}}(a)$. Then $a<b$.

2. For $\alpha=\psi_{\pi}^{\vec{\nu}}(a) \in O T, \max K(\vec{\nu}) \leq a$ holds.

Proof. 3.5ा1] Let $\beta=\psi_{\pi}^{\vec{\nu}}(b)$ with $\pi=\psi_{\kappa}^{\vec{\xi}}(a)$. Then $K_{\beta}(\{\pi, b\} \cup K(\vec{\nu}))<b$. On the other hand we have $\beta<\pi$. Hence $a \in K_{\beta}(\pi)<b$.

3.52 This is seen by induction on $\ell \alpha$. Ww have $c<a$ by Proposition 3.511 when $\pi=\psi_{\sigma}^{\vec{\mu}}(c)$

When $\alpha$ is constructed by Definition 3.12h, $\nu_{k}=m_{k}(\pi)+\Lambda^{m_{k+1}(\pi)} b$ holds for $b \leq a$. By IH we have $\max K(\vec{m}(\pi)) \leq c<a$ when $\pi=\psi_{\sigma}^{\vec{\mu}}(c)$.

Suppose $\alpha$ is constructed by Definition 3.121 We obtain $\vec{\nu}<_{s p} m_{2}(\pi)$, and hence $\max K(\vec{\nu}) \leq \max K\left(m_{2}(\pi)\right) \leq c<a$ by IH. 


\section{Operator controlled derivations}

In this section, operator controlled derivations are defined, which are introduced by W. Buchholz [6].

In this and the next sections except otherwise stated $\alpha, \beta, \gamma, \ldots, a, b, c, d, \ldots$ range over ordinal terms in $O T \subset \mathcal{H}_{\Lambda}(0), \xi, \zeta, \nu, \mu, \iota, \ldots$ range over ordinal terms in $E, \vec{\xi}, \vec{\zeta}, \vec{\nu}, \vec{\mu}, \vec{\iota}, \ldots$ range over finite sequences over ordinal terms in $E$, and $\pi, \kappa, \rho, \sigma, \tau, \lambda, \ldots$ range over regular ordinal terms $\mathbb{K}, \Omega_{\beta+1}, \psi_{\pi}^{\vec{\nu}}(a)$ with $\vec{\nu} \neq \overrightarrow{0}$. Reg denotes the set of regular ordinal terms. We write $\alpha \in \mathcal{H}_{a}(\beta)$ for $K_{\beta}(\alpha)<a$.

\subsection{Classes of sentences}

Following Buchholz [6] let us introduce a language for ramified set theory $R S$.

Definition 4.1 RS-terms and their levels are inductively defined.

1. For each $\alpha \in O T \cap \mathbb{K}, L_{\alpha}$ is an $R S$-term of level $\alpha$.

2. If $\phi\left(x, y_{1}, \ldots, y_{n}\right)$ is a set-theoretic formula in the language $\{\in\}$, and $a_{1}, \ldots, a_{n}$ are $R S$-terms of levels $<\alpha$, then $\left[x \in L_{\alpha}: \phi^{L_{\alpha}}\left(x, a_{1}, \ldots, a_{n}\right)\right]$ is an $R S$-term of level $\alpha$.

Each ordinal term $\alpha$ is denoted by the ordinal term $\left[x \in L_{\alpha}: x\right.$ is an ordinal], whose level is $\alpha$.

Definition $4.2 \quad 1 .|a|$ denotes the level of $R S$-terms $a$, and $\operatorname{Tm}(\alpha)$ the set of $R S$-terms of level $<\alpha . T m=T m(\mathbb{K})$ is then the set of $R S$-terms, which are denoted by $a, b, c, d, \ldots$

2. RS-formulas are constructed from literals $a \in b, a \notin b$ by propositional connectives $\vee, \wedge$, bounded quantifiers $\exists x \in a, \forall x \in a$ and unbounded quantifiers $\exists x, \forall x$. Unbounded quantifiers $\exists x, \forall x$ are denoted by $\exists x \in$ $L_{\mathbb{K}}, \forall x \in L_{\mathbb{K}}$, resp.

3. For $R S$-terms and $R S$-formulas $\iota, \mathrm{k}(\iota)$ denotes the set of ordinal terms $\alpha$ such that the constant $L_{\alpha}$ occurs in $\iota$.

4. For a set-theoretic $\Sigma_{n}$-formula $\psi\left(x_{1}, \ldots, x_{m}\right)$ in $\{\in\}$ and $a_{1}, \ldots, a_{m} \in$ $\operatorname{Tm}(\kappa), \psi^{L_{\kappa}}\left(a_{1}, \ldots, a_{m}\right)$ is a $\Sigma_{n}(\kappa)$-formula, where $n=0,1,2, \ldots$ and $\kappa \leq \mathbb{K} . \Pi_{n}(\kappa)$-formulas are defined dually.

5. For $\theta \equiv \psi^{L_{\kappa}}\left(a_{1}, \ldots, a_{m}\right) \in \Sigma_{n}(\kappa)$ and $\lambda<\kappa, \theta^{(\lambda, \kappa)}: \equiv \psi^{L_{\lambda}}\left(a_{1}, \ldots, a_{m}\right)$.

Note that the level $|t|=\max (\{0\} \cup \mathrm{k}(t))$ for $R S$-terms $t$. In what follows we need to consider sentences. Sentences are denoted $A, C$ possibly with indices.

The assignment of disjunctions and conjunctions to sentences is defined as in [6]. 
Definition 4.3 1. For $b, a \in T m(\mathbb{K})$ with $|b|<|a|$,

$$
(b \varepsilon a): \equiv \begin{cases}A(b) & \text { if } a \equiv\left[x \in L_{\alpha}: A(x)\right] \\ b \notin L_{0} & \text { if } a \equiv L_{\alpha}\end{cases}
$$

and $(a=b): \equiv(\forall x \in a(x \in b) \wedge \forall x \in b(x \in a))$.

2. For $b, a \in \operatorname{Tm}(\mathbb{K})$ and $J:=\operatorname{Tm}(|a|)$

$$
(b \in a): \simeq \bigvee(c \varepsilon a \wedge c=b)_{c \in J} \text { and }(b \notin a): \simeq \bigwedge(c \notin a \vee c \neq b)_{c \in J}
$$

3. $\left(A_{0} \vee A_{1}\right): \simeq \bigvee\left(A_{\iota}\right)_{\iota \in J}$ and $\left(A_{0} \wedge A_{1}\right): \simeq \bigwedge\left(A_{\iota}\right)_{\iota \in J}$ for $J:=2$.

4. For $a \in \operatorname{Tm}(\mathbb{K}) \cup\left\{L_{\mathbb{K}}\right\}$ and $J:=\operatorname{Tm}(|a|)$

$$
\exists x \in a A(x): \simeq \bigvee(b \varepsilon a \wedge A(b))_{b \in J} \text { and } \forall x \in a A(x): \simeq \bigwedge(b \notin k a \vee A(b))_{b \in J}
$$

The rank $\operatorname{rk}(\iota)$ of sentences or terms $\iota$ is defined as in [6].

Definition 4.4 1. $\operatorname{rk}(\neg A):=\operatorname{rk}(A)$.

2. $\operatorname{rk}\left(L_{\alpha}\right)=\omega \alpha$.

3. $\operatorname{rk}\left(\left[x \in L_{\alpha}: A(x)\right]\right)=\max \left\{\omega \alpha+1, \operatorname{rk}\left(A\left(L_{0}\right)\right)+2\right\}$.

4. $\operatorname{rk}(a \in b)=\max \{\operatorname{rk}(a)+6, \operatorname{rk}(b)+1\}$.

5. $\operatorname{rk}\left(A_{0} \vee A_{1}\right):=\max \left\{\operatorname{rk}\left(A_{0}\right), \operatorname{rk}\left(A_{1}\right)\right\}+1$.

6. $\operatorname{rk}(\exists x \in a A(x)):=\max \left\{\omega \operatorname{rk}(a), \operatorname{rk}\left(A\left(L_{0}\right)\right)+2\right\}$ for $a \in \operatorname{Tm}(\mathbb{K}) \cup\left\{L_{\mathbb{K}}\right\}$.

Proposition 4.5 Let $A$ be a sentence with $A \simeq \bigvee\left(A_{\iota}\right)_{\iota \in J}$ or $A \simeq \bigwedge\left(A_{\iota}\right)_{\iota \in J}$.

1. $\operatorname{rk}(A)<\mathbb{K}+\omega$.

2. $|A| \leq \operatorname{rk}(A) \in\{\omega|A|+i: i \in \omega\}$.

3. $\forall \iota \in J\left(\operatorname{rk}\left(A_{\iota}\right)<\operatorname{rk}(A)\right)$.

4. $\operatorname{rk}(A)<\lambda \Rightarrow A \in \Sigma_{0}(\lambda)$

\subsection{Operator controlled derivations}

By an operator we mean a map $\mathcal{H}, \mathcal{H}: \mathcal{P}(O T) \rightarrow \mathcal{P}(O T)$, such that

1. $\forall X \subset O T[X \subset \mathcal{H}(X)]$.

2. $\forall X, Y \subset O T[Y \subset \mathcal{H}(X) \Rightarrow \mathcal{H}(Y) \subset \mathcal{H}(X)]$. 
For an operator $\mathcal{H}$ and $\Theta, \Theta_{1} \subset O T, \mathcal{H}[\Theta](X):=\mathcal{H}(X \cup \Theta)$, and $\mathcal{H}[\Theta]\left[\Theta_{1}\right]:=$ $(\mathcal{H}[\Theta])\left[\Theta_{1}\right]$, i.e., $\mathcal{H}[\Theta]\left[\Theta_{1}\right](X)=\mathcal{H}\left(X \cup \Theta \cup \Theta_{1}\right)$.

Obviously $\mathcal{H}_{\alpha}$ is an operator for any $\alpha$, and if $\mathcal{H}$ is an operator, then so is $\mathcal{H}[\Theta]$.

Sequents are finite sets of sentences, and inference rules are formulated in one-sided sequent calculus. Let $\mathcal{H}=\mathcal{H}_{\gamma}(\gamma \in O T)$ be an operator, $\Theta$ a finite set of $\mathbb{K}, \Gamma$ a sequent, $a \in O T$ and $b \in O T \cap(\mathbb{K}+\omega)$.

We define a relation $\left(\mathcal{H}_{\gamma}, \Theta\right) \vdash_{b}^{a} \Gamma$, which is read there exists an infinitary derivation of $\Gamma$ which is $\Theta$-controlled by $\mathcal{H}_{\gamma}$, and whose height is at most $a$ and its cut rank is less than $b$ '.

$\kappa, \lambda, \sigma, \tau, \pi$ ranges over regular ordinal terms.

Definition $4.6\left(\mathcal{H}_{\gamma}, \Theta\right) \vdash_{b}^{a} \Gamma$ holds if

$$
\mathrm{k}(\Gamma) \cup\{a\} \subset \mathcal{H}_{\gamma}[\Theta]
$$

and one of the following cases holds:

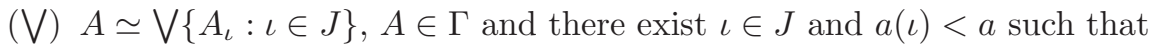

$$
|\iota|<a
$$

and $\left(\mathcal{H}_{\gamma}, \Theta\right) \vdash_{b}^{a(\iota)} \Gamma, A_{\iota}$.

$(\bigwedge) A \simeq \bigwedge\left\{A_{\iota}: \iota \in J\right\}, A \in \Gamma$ and for every $\iota \in J$ there exists an $a(\iota)<a$ such that $\left(\mathcal{H}_{\gamma}, \Theta \cup\{\mathrm{k}(\iota)\}\right) \vdash_{b}^{a(\iota)} \Gamma, A_{\iota}$.

(cut) There exist $a_{0}<a$ and $C$ such that $\operatorname{rk}(C)<b$ and $\left(\mathcal{H}_{\gamma}, \Theta\right) \vdash_{b}^{a_{0}} \Gamma, \neg C$ and $\left(\mathcal{H}_{\gamma}, \Theta\right) \vdash_{b}^{a_{0}} C, \Gamma$.

$\left(\Omega \in M_{2}\right)$ There exist ordinals $a_{\ell}, a_{r}(\alpha)$ and a sentence $C \in \Pi_{2}(\Omega)$ such that $\sup \left\{a_{\ell}+1, a_{r}(\alpha)+1: \alpha<\Omega\right\} \leq a, b \geq \Omega,\left(\mathcal{H}_{\gamma}, \Theta\right) \vdash_{b}^{a_{\ell}} \Gamma, C$ and $\left(\mathcal{H}_{\gamma}, \Theta \cup\right.$ $\{\omega \alpha\}) \vdash_{b}^{a_{r}(\alpha)} \neg C^{(\alpha, \Omega)}, \Gamma$ for any $\alpha<\Omega$.

$(\operatorname{rfl}(\pi, k, \vec{\xi}, \vec{\nu}))$ There exist a Mahlo ordinal $\mathbb{K} \geq \pi \in \mathcal{H}_{\gamma}[\Theta] \cap(b+1)$, an integer $2 \leq k \leq N$ and sequences $\vec{\nu}=\left(\nu_{2}, \ldots, \nu_{N-1}\right), \vec{\xi}=\left(\xi_{2}, \ldots, \xi_{N-1}\right) \in S D$ of ordinals $\nu_{i}, \xi_{i} \in E$, ordinals $a_{\ell}, a_{r}(\rho), a_{0}$, and a finite set $\Delta$ of $\Sigma_{k}(\pi)$ sentences enjoying the following conditions: When $\pi=\mathbb{K}, k=N$ and $\vec{\nu}=\overrightarrow{0}$ with $\operatorname{lh}(\vec{\nu})=N-1$ hold. Also let $\vec{\xi}=\overrightarrow{0}$ in this case. When $\pi<\mathbb{K}$, $\xi_{k} \neq 0$ with $k<N, \overrightarrow{0} \neq \vec{\xi}$, and $\forall i\left(\xi_{i} \leq_{s p} m_{i}(\pi)\right)$.

1. When $\pi<\mathbb{K}$, cf. Definitions 2.119,

$$
\forall i<k\left(\nu_{i}=\xi_{i}\right) \&\left(\nu_{k}, \ldots, \nu_{N-1}\right)<_{s d} \xi_{k} \& K(\vec{\nu}) \cup K(\vec{\xi}) \subset \mathcal{H}_{\gamma}[\Theta]
$$

and

$$
\forall \mu \in \vec{\nu} \cup \vec{\xi} \cup \vec{m}(\pi)\left(K(\mu) \subset \mathcal{H}_{\max K(\mu)}[\Theta]\right)
$$

cf. (4). 
2. For each $\delta \in \Delta,\left(\mathcal{H}_{\gamma}, \Theta\right) \vdash_{b}^{a_{\ell}} \Gamma, \neg \delta$.

3. $H(\vec{\nu}, \pi, \gamma, \Theta)$ denotes the resolvent class for $\vec{\nu}, \pi, \gamma$ and $\Theta$ defined as follows:

$$
\begin{aligned}
& C(\pi, \gamma, \Theta) \quad:=\left\{\rho<\pi: \mathcal{H}_{\gamma}(\rho) \cap \pi \subset \rho \& \Theta \cap \pi \subset \rho\right\} \\
& \rho \in H(\vec{\nu}, \pi, \gamma, \Theta) \quad: \Leftrightarrow \quad \forall i\left(\nu_{i} \leq_{s p} m_{i}(\rho) \wedge K\left(m_{i}(\rho)\right) \subset \mathcal{H}_{\max K\left(m_{i}(\rho)\right)}(\rho)\right) \\
& \text { for } \rho \in \operatorname{Reg} \cap C(\pi, \gamma, \Theta) \text {. } \\
& \text { Then for each } \rho \in H(\vec{\nu}, \pi, \gamma, \Theta),\left(\mathcal{H}_{\gamma}, \Theta \cup\{\rho\}\right) \vdash_{b}^{a_{r}(\rho)} \Gamma, \Delta^{(\rho, \pi)} \text {. }
\end{aligned}
$$

4 .

$$
\sup \left\{a_{\ell}, a_{r}(\rho): \rho \in H(\vec{\nu}, \pi, \gamma, \Theta)\right\} \leq a_{0} \in \mathcal{H}_{\gamma}[\Theta] \cap a
$$

In the inference rule $(\operatorname{rfl}(\pi, k, \vec{\xi}, \vec{\nu}))$ for $\pi=\psi_{\sigma}^{\vec{\xi}}(c)<\mathbb{K}$, we have $\pi \in$ $M h_{2}^{c}(\vec{\xi})$. In particular, $\pi \in \bigcap_{i<k} M h_{i}^{c}\left(\xi_{i}\right) \cap M h_{k}^{c}\left(\xi_{k}\right)$. Also we are assuming $\left(\nu_{k}, \ldots, \nu_{N-1}\right)<_{s d} \xi_{k}$, a fortiori $\left(\nu_{k}, \ldots, \nu_{N-1}\right)<\xi_{k}$. Since $\pi \in \bigcap_{i<k} M h_{i}^{c}\left(\nu_{i}\right)$ is a $\Pi_{k}$-sentence holding on $L_{\pi}$, we obtain $\pi \in M_{k}\left(M h_{2}^{c}(\vec{\nu})\right)$. Thus the reflection rule $(\operatorname{rfl}(\pi, k, \vec{\nu}))$ says that $\pi$ is $\Pi_{k}$-reflecting on the class $H\left(\vec{\nu}, \pi, \gamma, \gamma_{0}, \Theta\right)$ for the club subset $C(\pi, \gamma, \Theta)$ of $\pi$, cf. Proposition 2.13. On the other side we see $\rho \in M h_{2}^{a}(\vec{\nu})$ from Proposition 2.9 if $\forall i\left(\nu_{i} \leq m_{i}(\rho)\right)$ for $\rho \in M h_{2}^{a}(\vec{m}(\rho))$.

We will state some lemmas for the operator controlled derivations. These can be shown as in [6]. In what follows by an operator $\mathcal{H}$ we mean an $\mathcal{H}_{\gamma}$ for an ordinal $\gamma$.

Lemma 4.7 Let $\left(\mathcal{H}_{\gamma}, \Theta\right) \vdash_{b}^{a} \Gamma$.

1. $\left(\mathcal{H}_{\gamma^{\prime}}, \Theta \cup \Theta_{0}\right) \vdash_{b^{\prime}}^{a^{\prime}} \Gamma, \Delta$ for any $\gamma^{\prime} \geq \gamma$, any $\Theta_{0}$, and any $a^{\prime} \geq a, b^{\prime} \geq b$ such that $\mathrm{k}(\Delta) \cup\left\{a^{\prime}\right\} \subset \mathcal{H}_{\gamma^{\prime}}\left[\Theta \cup \Theta_{0}\right]$.

2. Assume $\Theta_{1} \cup\{c\}=\Theta, c \in \mathcal{H}_{\gamma}\left[\Theta_{1}\right]$. Then $\left(\mathcal{H}_{\gamma}, \Theta_{1}\right) \vdash_{b}^{a} \Gamma$.

Lemma 4.8 (Tautology) $(\mathcal{H}, \mathrm{k}(\Gamma \cup\{A\})) \vdash_{0}^{2 \operatorname{rk}(A)} \Gamma, \neg A, A$.

Lemma 4.9 (Inversion) Let $A \simeq \bigwedge\left(A_{\iota}\right)_{\iota \in J}$, and $(\mathcal{H}, \Theta) \vdash_{b}^{a} \Gamma$ with $A \in \Gamma$. Then for any $\iota \in J,(\mathcal{H}, \Theta \cup \mathrm{k}(\iota)) \vdash_{b}^{a} \Gamma, A_{\iota}$ holds.

Lemma 4.10 (Boundedness) Suppose $(\mathcal{H}, \Theta) \vdash_{c}^{a} \Gamma, C$ for $a C \in \Sigma_{1}(\lambda)$, and $a \leq b \in \mathcal{H} \cap \lambda$. Then $(\mathcal{H}, \Theta) \vdash_{c}^{a} \Gamma, C^{(b, \lambda)}$.

Lemma 4.11 (Persistency) Suppose $(\mathcal{H}, \Theta) \vdash_{c}^{a} \Gamma, C^{(b, \lambda)}$ for $a C \in \Sigma_{1}(\lambda)$ and $a b<\lambda \in \mathcal{H}[\Theta]$. Then $(\mathcal{H}, \Theta) \vdash_{c}^{a} \Gamma, C$.

Lemma 4.12 (Predicative Cut-elimination) Suppose $(\mathcal{H}, \Theta) \vdash_{c+\omega^{a}}^{b} \Gamma, a \in \mathcal{H}[\Theta]$ and $\left.] c, c+\omega^{a}\right] \cap \operatorname{Reg}=\emptyset$. Then $(\mathcal{H}, \Theta) \vdash_{c}^{\varphi a b} \Gamma$.

Lemma 4.13 (Embedding of Axioms)

For each axiom $A$ in $\mathrm{KP}_{N}$, there is an $m<\omega$ such that for any operator $\mathcal{H}=\mathcal{H}_{\gamma},(\mathcal{H}, \emptyset) \vdash \mathbb{K} \cdot 2$. $A$ holds. 
Proof. The axiom $\neg A, \exists z A^{(z)}$ for $\Pi_{N}$-reflection follows from $A, \neg A$ and $\exists z A^{(z)}, \neg A^{(\rho)}$ for regular ordinals $\rho<\mathbb{K}$ by an inference $(\mathrm{rfl}(\mathbb{K}, N, \overrightarrow{0}, \overrightarrow{0}))$.

Lemma 4.14 (Embedding) If $\mathrm{KP}_{N} \vdash \Gamma$ for sets $\Gamma$ of sentences, there are $m, k<\omega$ such that for any operator $\mathcal{H}=\mathcal{H}_{\gamma},(\mathcal{H}, \emptyset) \vdash_{\mathbb{K}+m}^{\mathbb{K} \cdot 2+k} \Gamma$ holds

\section{Lowering and eliminating higher Mahlo oper- ations}

In the section inferences $(\mathrm{rff}(\mathbb{K}, N, \overrightarrow{0}, \overrightarrow{0}))$ for $\Pi_{N}$-reflecting ordinals $\mathbb{K}$ are eliminated from operator controlled derivations of $\Sigma_{1}$-sentences $\varphi^{L_{\Omega}}$ over $\Omega$.

$\alpha \# \beta$ denotes the natural (commutative) sum of ordinal terms $\alpha, \beta$.

Lemma 5.1 For a Mahlo term $\pi \in O T, \vec{\xi} \in S D$ denotes a sequence with $\operatorname{lh}(\vec{\xi})=N-2$, and $2 \leq k \leq N-1$ an integer for which the following hold: When $\pi=\mathbb{K}$, let $\vec{\xi}=\overrightarrow{0}$ and $k=N-1$. Otherwise $\vec{\xi}=\left(\xi_{2}, \ldots, \xi_{k+1}\right) * \overrightarrow{0}$ with $\xi_{k+1} \neq 0$ such that $\forall i \leq k+1\left(\xi_{i} \leq_{s p} m_{i}(\pi)\right)$.

For ordinal terms $\gamma, a \in O T$ let us define a sequence $\vec{\zeta}(a):=\left(\zeta_{2}(a), \ldots, \zeta_{k}(a)\right) *$ $\overrightarrow{0}$ with $\operatorname{lh}(\vec{\zeta}(a))=N-2$ as follows. $\vec{\zeta}(a)=\overrightarrow{0} *(\gamma+a)$ when $\pi=\mathbb{K}$. Otherwise $\zeta_{k}(a)=\xi_{k}+\Lambda^{\xi_{k+1}}(\gamma+a)$ and $\zeta_{i}(a)=\xi_{i}$ for $i<k$.

Let $\kappa \in H(\vec{\zeta}(a), \pi, \gamma, \Theta)$ for a finite set $\Theta \subset O T$.

Now suppose $\left(\mathcal{H}_{\gamma}, \Theta\right) \vdash_{\pi}^{a} \Gamma$ where $\{\gamma, \pi\} \cup K(\vec{\xi}) \subset \mathcal{H}_{\gamma}[\Theta], \Theta \subset \pi, \forall i\left(K\left(\xi_{i}\right) \subset\right.$ $\left.\mathcal{H}_{\max K\left(\xi_{i}\right)}[\Theta]\right)$, and $\Gamma \subset \Pi_{k+1}(\pi)$.

Let $\gamma(a, b)=\gamma \# a \# b, \beta(a, b)=\psi_{\pi}(\gamma(a, b))$, and $c>\gamma(a, \kappa)$. Then the following holds:

$$
\left(\mathcal{H}_{c}, \Theta \cup\{\kappa\}\right) \vdash_{\kappa}^{\beta(a, \kappa)} \Gamma^{(\kappa, \pi)}
$$

Proof by induction on $a$. Let $\kappa \in H(\vec{\zeta}(a), \pi, \gamma, \Theta)$. We see $\vec{\zeta}(a) \in S D$, and from (5) and $\Theta \subset \kappa$ that

$$
\mathrm{k}(\Gamma) \cap \pi \subset \mathcal{H}_{\gamma}(\kappa) \cap \pi \subset \kappa
$$

For any $a \in \mathcal{H}_{\gamma}[\Theta]$, we obtain $\{\gamma, \pi, a, \kappa\} \subset \mathcal{H}_{\gamma}(\pi)$ by $\Theta \cup\{\kappa\} \subset \pi$. Hence for $\gamma(a, \kappa)=\gamma \# a \# \kappa,\{\gamma(a, \kappa), \pi\} \subset \mathcal{H}_{\gamma}(\pi)$, and $\{\gamma(a, \kappa), \pi\} \subset \mathcal{H}_{\gamma(a, \kappa)}(\beta(a, \kappa))$ by the definition (3). Therefore $\kappa \in \mathcal{H}_{\gamma(a, \kappa)}(\beta(a, \kappa)) \cap \pi \subset \beta(a, \kappa)$ by Proposition 2.6] and $\Theta \subset \beta(a, \kappa)<\pi$. Thus we obtain

$$
\left\{a_{0}, a_{1}\right\} \subset \mathcal{H}_{\gamma}\left[\Theta \cup \Theta_{0}\right] \& a_{0}<a_{1} \& \Theta_{0} \subset \kappa \Rightarrow \beta\left(a_{0}, \kappa\right)<\beta\left(a_{1}, \kappa\right) .
$$

Case 1. First consider the case when the last inference is a $(\operatorname{rfl}(\pi, k+1, \vec{\xi}, \vec{\nu}))$.

We have $a_{\ell} \in \mathcal{H}_{\gamma}[\Theta] \cap a, a_{r}(\rho) \in \mathcal{H}_{\gamma}[\Theta \cup\{\rho\}] \cap a$, and a finite set $\Delta$ of $\Sigma_{k+1}(\pi)$-sentences. We have for each $\delta \in \Delta$

$$
\left(\mathcal{H}_{\gamma}, \Theta\right) \vdash_{\pi}^{a_{\ell}} \Gamma, \neg \delta
$$


and for each $\rho \in H(\vec{\nu}, \pi, \gamma, \Theta)$

$$
\left(\mathcal{H}_{\gamma}, \Theta \cup\{\rho\}\right) \vdash_{\pi}^{a_{r}(\rho)} \Gamma, \Delta^{(\rho, \pi)}
$$

When $\pi<\mathbb{K}, \vec{\nu}=\left(\nu_{2}, \ldots, \nu_{N-1}\right) \in S D$ is a sequence such that $\forall i<$ $k+1\left(\nu_{i}=\xi_{i}\right),\left(\nu_{k+1}, \ldots, \nu_{N-1}\right)<_{s d} \xi_{k+1}, K(\vec{\nu}) \cup K(\vec{\xi}) \subset \mathcal{H}_{\gamma}[\Theta]$, and $\forall i\left(K\left(\nu_{i}\right) \subset\right.$ $\left.\mathcal{H}_{\max K\left(\nu_{i}\right)}[\Theta]\right)$, cf. (77) and (8).

Let $\Gamma_{0}=\Gamma \cap \Sigma_{k}(\pi)$ and $\left\{\forall x \in L_{\pi} \theta_{i}(x): i=1, \ldots, n\right\}(n \geq 0)=\Gamma \backslash \Gamma_{0}$ for $\Sigma_{k}(\pi)$-formulas $\theta_{i}(x)$. Let us fix $\vec{d}=\left\{d_{1}, \ldots, d_{n}\right\} \subset T m(\kappa)$ arbitrarily. Put $\mathrm{k}(\vec{d})=\bigcup\left\{\mathrm{k}\left(d_{i}\right): i=1, \ldots, n\right\}$ and $\Gamma(\vec{d})=\Gamma_{0} \cup\left\{\theta_{i}\left(d_{i}\right): i=1, \ldots, n\right\}$.

By Inversion lemma 4.9 from (13) we obtain for each $\delta \in \Delta$

$$
\left(\mathcal{H}_{\gamma}, \Theta \cup \mathrm{k}(\vec{d})\right) \vdash_{\pi}^{a_{\ell}} \Gamma(\vec{d}), \neg \delta
$$

Let $\rho \in C(\kappa, c, \Theta \cup\{\kappa\} \cup \mathrm{k}(\vec{d}))$. We see $\rho<\kappa$, and $\mathrm{k}(\vec{d})<\rho$ from $\mathrm{k}(\vec{d})<\kappa$. By $\Theta \cap \pi \subset \mathcal{H}_{\gamma}(\kappa) \cap \pi \subset \kappa$ and $\gamma \leq c$ we obtain $C(\kappa, c, \Theta \cup\{\kappa\} \cup \mathrm{k}(\vec{d})) \subset C(\pi, \gamma, \Theta)$. Namely, cf. (9)

$$
\rho \in H(\vec{\nu}, \kappa, c, \Theta \cup\{\kappa\} \cup \mathrm{k}(\vec{d})) \Rightarrow \rho \in H(\vec{\nu}, \pi, \gamma, \Theta)
$$

For each $\rho \in H(\vec{\nu}, \kappa, c, \Theta \cup\{\kappa\} \cup \mathrm{k}(\vec{d}))$, IH with (14) and (16) yields for $c>$ $\gamma\left(a_{r}(\rho), \kappa\right)$ and $\kappa \in H\left(\vec{\zeta}\left(a_{r}(\rho)\right), \pi, \gamma, \Theta \cup\{\rho\}\right)$

$$
\left(\mathcal{H}_{c}, \Theta \cup\{\rho, \kappa\}\right) \vdash_{\kappa}^{\beta\left(a_{r}(\rho), \kappa\right)} \Gamma^{(\kappa, \pi)}, \Delta^{(\rho, \pi)}
$$

Let $\rho \in M_{\ell}:=\left\{\rho \in \operatorname{Reg}: \forall i\left(\zeta_{i}\left(a_{\ell}\right) \leq_{s p} m_{i}(\rho)\right)\right\} \cap H(\vec{\nu}, \kappa, c, \Theta \cup\{\kappa\} \cup \mathrm{k}(\vec{d}))$. Then $M_{\ell} \subset H\left(\vec{\zeta}\left(a_{\ell}\right), \pi, \gamma, \Theta \cup \mathrm{k}(\vec{d})\right)$ and $\Theta \cup \mathrm{k}(\vec{d}) \subset \rho$. For each $\delta \in \Delta$, IH with (15) yields for $c>\gamma\left(a_{\ell}, \rho\right)$

$$
\left(\mathcal{H}_{c}, \Theta \cup \mathrm{k}(\vec{d}) \cup\{\rho\}\right) \vdash_{\rho}^{\beta\left(a_{\ell}, \rho\right)} \Gamma(\vec{d})^{(\rho, \pi)}, \neg \delta^{(\rho, \pi)}
$$

From (17) and (18) by several (cut)'s of $\delta^{(\rho, \pi)}$ with $\operatorname{rk}\left(\delta^{(\rho, \pi)}\right)<\kappa$ we obtain for $a(\rho)=\max \left\{a_{\ell}, a_{r}(\rho)\right\}$ and some $p<\omega$

$$
\left\{\left(\mathcal{H}_{c}, \Theta \cup \mathrm{k}(\vec{d}) \cup\{\kappa, \rho\}\right) \vdash_{\kappa}^{\beta(a(\rho), \kappa)+p} \Gamma(\vec{d})^{(\rho, \pi)}, \Gamma^{(\kappa, \pi)}: \rho \in M_{\ell}\right\}
$$

On the other hand we have by Tautology lemma 4.8 for each $\theta(\vec{d})^{(\kappa, \pi)} \in \Gamma(\vec{d})^{(\kappa, \pi)}$

$$
\left(\mathcal{H}_{\gamma}, \Theta \cup \mathrm{k}(\vec{d}) \cup\{\kappa\}\right) \vdash_{0}^{2 \operatorname{rk}\left(\theta(\vec{d})^{(\kappa, \pi)}\right)} \Gamma(\vec{d})^{(\kappa, \pi)}, \neg \theta(\vec{d})^{(\kappa, \pi)}
$$

where $2 \operatorname{rk}\left(\theta(\vec{d})^{(\kappa, \pi)}\right) \leq \kappa+p$ for some $p<\omega$.

Moreover we have $\sup \left\{2 \operatorname{rk}\left(\theta(\vec{d})^{(\kappa, \pi)}\right), \beta(a(\rho), \kappa)+p: \rho \in M_{\ell}\right\} \leq \beta\left(a_{0}, \kappa\right)+$ $p \in \mathcal{H}_{\gamma}[\Theta \cup\{\kappa\}]$, where $\sup \left\{a_{\ell}, a_{r}(\rho): \rho \in H(\vec{\nu}, \pi, \gamma, \Theta)\right\} \leq a_{0}<a$ by (10).

Now let $\vec{\mu}=\left(\mu_{2}, \ldots, \mu_{N-1}\right)=\max \left\{\vec{\zeta}\left(a_{\ell}\right), \vec{\nu}\right\}$ with $\mu_{i}=\max \left\{\zeta_{i}\left(a_{\ell}\right), \nu_{i}\right\}$. Since $\nu_{i}=\xi_{i} \leq_{p t} \zeta_{i}\left(a_{\ell}\right)$ for $i<k+1$, we obtain $\mu_{i}=\left\{\begin{array}{ll}\zeta_{i}\left(a_{\ell}\right) & i \leq k \\ \nu_{i} & i>k\end{array}\right.$. We see 
that $M_{\ell}=H(\vec{\mu}, \kappa, c, \Theta \cup\{\kappa\} \cup \mathrm{k}(\vec{d}))$. Moreover we have $\forall i<k\left(\mu_{i}=\xi_{i}=\zeta_{i}(a)\right)$ and $\left(\mu_{k}, \ldots, \mu_{N-1}\right)=\left(\zeta_{k}\left(a_{\ell}\right)\right) *\left(\nu_{k+1}, \ldots, \nu_{N-1}\right)<_{s d} \zeta_{k}(a)$. Also $\forall i\left(K\left(\zeta_{i}(a)\right) \subset\right.$ $\left.\mathcal{H}_{\max K\left(\zeta_{i}(a)\right)}[\Theta]\right)$ and $\forall i\left(K\left(\mu_{i}\right) \subset \mathcal{H}_{\max K\left(\mu_{i}\right)}[\Theta]\right)$. For $\neg \Gamma(\vec{d})^{(\kappa, \pi)} \subset \Pi_{k}(\kappa)$, by an inference rule $(\operatorname{rfl}(\kappa, k, \vec{\zeta}(a), \vec{\mu}))$ with its resolvent class $M_{\ell}$, we conclude from (20) and (19) that $\left(\mathcal{H}_{c}, \Theta \cup\{\kappa\} \cup \mathrm{k}(\vec{d})\right) \vdash_{\kappa}^{\beta\left(a_{0}, \kappa\right)+p+1} \Gamma(\vec{d})^{(\kappa, \pi)}, \Gamma^{(\kappa, \pi)}$. Since $\vec{d} \subset \operatorname{Tm}(\kappa)$ is arbitrary, several $(\bigwedge)$ 's yield (11).

Case 2. Second consider the case when the last inference is a $(\operatorname{rfl}(\pi, j, \vec{\xi}, \vec{\nu}))$ for a $j<k+1$. We have $\left(\mathcal{H}_{\gamma}, \Theta\right) \vdash_{\pi}^{a_{\ell}} \Gamma, \neg \delta$ for each $\delta \in \Delta \subset \Sigma_{j}(\pi)$ with $a_{\ell} \in \mathcal{H}_{\gamma}[\Theta] \cap a$, and $\left(\mathcal{H}_{\gamma}, \Theta \cup\{\rho\}\right) \vdash_{\pi}^{a_{r}(\rho)} \Gamma, \Delta^{(\rho, \pi)}$ for each $\rho \in H(\vec{\nu}, \pi, \gamma, \Theta)$ with $a_{r}(\rho) \in \mathcal{H}_{\gamma}[\Theta \cup\{\rho\}] \cap a . \vec{\nu} \in S D$ is a sequence such that $\forall i<j\left(\nu_{i}=\xi_{i}\right)$ and $\left(\nu_{j}, \ldots, \nu_{N-1}\right)<_{s d} \xi_{j}$.

We see that the resolvent class $H\left(\vec{\nu}, \kappa, c_{1}, \Theta \cup\{\kappa\}\right)$ is a subclass of $H(\vec{\nu}, \pi, \gamma, \Theta)$. By IH we have $\left(\mathcal{H}_{c}, \Theta \cup\{\kappa\}\right) \vdash_{\kappa}^{\beta\left(a_{\ell}, \kappa\right)} \Gamma^{(\kappa, \pi)}, \neg \delta^{(\kappa, \pi)}$ for each $\delta \in \Delta$, and $\left(\mathcal{H}_{c}, \Theta \cup\{\kappa, \rho\}\right) \vdash_{\kappa}^{\beta\left(a_{r}(\rho), \kappa\right)} \Gamma^{(\kappa, \pi)}, \Delta^{(\rho, \pi)}$ for each $\rho \in H(\vec{\nu}, \kappa, c, \Theta \cup\{\kappa\})$ with $\Delta^{(\rho, \pi)}=\left(\Delta^{(\kappa, \pi)}\right)^{(\rho, \kappa)}$. We claim that $\forall i \leq j\left(\xi_{j} \leq_{s p} m_{i}(\kappa)\right)$. Consider the case when $i=j=k$. Then we have $\xi_{k} \leq_{s p} m_{k}(\pi)$ and $\zeta_{k}(a) \leq_{s p} m_{k}(\kappa)$ with $\xi_{k}<_{p t} \zeta_{k}(a)$. We obtain $\xi_{k} \leq_{s p} m_{k}(\kappa)$. Hence by an inference rule $(\operatorname{rfl}(\kappa, j, \vec{\xi}(j), \vec{\nu}))$ for the sequence $\vec{\xi}(j)=\left(\xi_{2}, \ldots, \xi_{j}\right) * \overrightarrow{0} \in S D$, cf. Proposition 2.211, we obtain (11).

Case 3. Third consider the case when the last inference is a $(\operatorname{rfl}(\sigma, j, \vec{\mu}, \vec{\nu}))$ for a $\sigma<\pi$. We have $\left(\mathcal{H}_{\gamma}, \Theta\right) \vdash_{\pi}^{a_{\ell}} \Gamma, \neg \delta$ for each $\delta \in \Delta \subset \Sigma_{j}(\sigma)$, and $\left(\mathcal{H}_{\gamma}, \Theta \cup\right.$ $\{\rho\}) \vdash_{\pi}^{a_{r}(\rho)} \Gamma, \Delta^{(\rho, \sigma)}$ for each $\rho \in H(\vec{\nu}, \sigma, \gamma, \Theta)$. We obtain $\sigma<\kappa$ by (12) for $\sigma \in \mathcal{H}_{\gamma}[\Theta]$. Hence $\Delta \subset \Sigma_{0}^{1}(\sigma) \subset \Sigma_{0}(\kappa)$ and $\delta^{(\kappa, \pi)} \equiv \delta$ for any $\delta \in \Delta$. Let $H(\vec{\nu}, \sigma, c, \Theta \cup\{\kappa\})$ be the resolvent class for $\sigma, \vec{\nu}, c$ and $\Theta \cup\{\kappa\}$. Then $H(\vec{\nu}, \sigma, c, \Theta \cup\{\kappa\}) \subset H(\vec{\nu}, \sigma, \gamma, \Theta)$.

From IH we have $\left(\mathcal{H}_{c}, \Theta \cup\{\kappa\}\right) \vdash_{\kappa}^{\beta\left(a_{\ell}, \kappa\right)} \Gamma^{(\kappa, \pi)}, \neg \delta$ for each $\delta \in \Delta$, and $\left(\mathcal{H}_{c}, \Theta \cup\{\kappa, \rho\}\right) \vdash_{\kappa}^{\beta\left(a_{r}(\rho), \kappa\right)} \Gamma^{(\kappa, \pi)}, \Delta^{(\rho, \sigma)}$ for each $\rho \in H(\vec{\nu}, \sigma, c, \Theta \cup\{\kappa\})$. We obtain (11) by an inference rule $(\mathrm{rfl}(\sigma, j, \vec{\mu}, \vec{\nu}))$ with the resolvent class $H(\vec{\nu}, \sigma, c, \Theta \cup$ $\{\kappa\})$.

Case 4. Fourth consider the case when the last inference $(\bigwedge)$ introduces a $\Pi_{k+1}(\pi)$-sentence $\left(\forall x \in L_{\pi} \theta(x)\right) \in \Gamma$. We have $\left(\mathcal{H}_{\gamma}, \Theta \cup \mathrm{k}(d)\right) \vdash_{\pi}^{a(d)} \Gamma, \theta(d)$ for each $d \in T m(\pi)$. For each $d \in T m(\kappa)$, IH with $\mathrm{k}(d)<\kappa$ yields $\left(\mathcal{H}_{c}, \Theta \cup\{\kappa\} \cup\right.$ $\mathrm{k}(d)) \vdash_{\kappa}^{\beta(a(d), \kappa)} \Gamma^{(\kappa, \pi)}, \theta(d)^{(\kappa, \pi)}$. (\) yields (11) for $\forall x \in L_{\kappa} \theta(x)^{(\kappa, \pi)} \equiv(\forall x \in$ $\left.L_{\pi} \theta(x)\right)^{(\kappa, \pi)} \in \Gamma^{(\kappa, \pi)}$.

Case 5. Fifth consider the case when the last inference $(\bigwedge)$ introduces a $\Sigma_{0}(\pi)$ sentence $(\forall x \in c \theta(x)) \in \Gamma$ for a $c \in T m(\pi)$. We have $\left(\mathcal{H}_{\gamma}, \Theta \cup \mathrm{k}(d)\right) \vdash_{\pi}^{a(d)} \Gamma, \theta(d)$ for each $d \in \operatorname{Tm}(|c|)$. Then we have $|d|<|c|<\kappa$ by (12). IH yields $\left(\mathcal{H}_{c}, \Theta \cup\{\kappa\} \cup \mathrm{k}(d) \vdash_{\kappa}^{\beta(a(d), \kappa)} \Gamma^{(\kappa, \pi)}, \theta(d)\right.$, and we obtain (11) by an inference $(\bigwedge)$. 
Case 6. Sixth consider the case when the last inference $(\bigvee)$ introduces a $\Sigma_{k}(\pi)$ sentence $\left(\exists x \in L_{\pi} \theta(x)\right) \in \Gamma$. We have $\left(\mathcal{H}_{\gamma}, \Theta\right) \vdash_{\pi}^{a_{0}} \Gamma, \theta(d)$ for a $d \in T m(\pi)$. Without loss of generality we can assume that $\mathrm{k}(d) \subset \mathrm{k}(\theta(d))$. Then we see that $|d|<\kappa$ from (12), and $d \in \operatorname{Tm}(\kappa)$. Also $|d|<\kappa<\beta(a, \kappa)$ for (6). IH yields with $\left(\exists x \in L_{\pi} \theta(x)\right)^{(\kappa, \pi)} \equiv\left(\exists x \in L_{\kappa} \theta(x)^{(\kappa, \pi)}\right) \in \Gamma^{(\kappa, \pi)},\left(\mathcal{H}_{c}, \Theta \cup\{\kappa\}\right) \vdash_{\kappa}^{\beta\left(a_{0}, \kappa\right)}$ $\Gamma^{(\kappa, \pi)}, \theta(d)^{(\kappa, \pi)}$, and we obtain (11) by an inference $(\bigvee)$.

Case 7. Seventh consider the case when the last inference is a (cut). We have $\left(\mathcal{H}_{\gamma}, \Theta\right) \vdash_{\pi}^{a_{0}} \Gamma, \neg C$ and $\left(\mathcal{H}_{\gamma}, \Theta\right) \vdash_{\pi}^{a_{0}} C, \Gamma$ for $a_{0}<a$ with $\operatorname{rk}(C)<\pi$. Then $C \in \Sigma_{0}(\pi)$ by Proposition 4.54 On the other side $\mathrm{k}(C) \subset \pi$ holds by Proposition 4.512, Then $\mathrm{k}(C) \subset \kappa$ by (12). Hence $C^{(\kappa, \pi)} \equiv C$ and $\operatorname{rk}\left(C^{(\kappa, \pi)}\right)<\kappa$ again by Proposition 4.512. IH yields $\left(\mathcal{H}_{c}, \Theta \cup\{\kappa\}\right) \vdash_{\kappa}^{\beta\left(a_{0}, \kappa\right)} \Gamma^{(\kappa, \pi)}, \neg C^{(\kappa, \pi)}$ and $\left(\mathcal{H}_{c}, \Theta \cup\{\kappa\}\right) \vdash_{\kappa}^{\beta\left(a_{0}, \kappa\right)} C^{(\kappa, \pi)}, \Gamma^{(\kappa, \pi)}$. Hence by a $(c u t)$ we obtain (11).

Case 8. Eighth consider the case when the last inference is an $\left(\Omega \in M_{2}\right)$. We have $\left(\mathcal{H}_{\gamma}, \Theta\right) \vdash_{\pi}^{a_{\ell}} \Gamma, C$ and $\left(\mathcal{H}_{\gamma}, \Theta \cup\{\omega \alpha\}\right) \vdash_{\pi}^{a_{r}(\alpha)} \neg C^{(\alpha, \Omega)}, \Gamma$ for each $\alpha<\Omega$ with $\sup \left\{a_{\ell}+1, a_{r}(\alpha)+1: \alpha<\Omega\right\} \leq a$ and $C \in \Pi_{2}(\Omega)$.

We obtain $\omega \alpha<\kappa$ for $\alpha<\Omega$. IH with $C^{(\kappa, \pi)} \equiv C$ yields for each $\alpha<\Omega$, $\left(\mathcal{H}_{c}, \Theta \cup\{\kappa, \omega \alpha\}\right) \vdash_{\kappa}^{\beta\left(a_{r}(\alpha), \kappa\right)} \neg C^{(\alpha, \Omega)}, \Gamma^{(\kappa, \pi)}$, and $\left(\mathcal{H}_{c}, \Theta \cup\{\kappa\}\right) \vdash_{\kappa}^{\beta\left(a_{\ell}, \kappa\right)} \Gamma^{(\kappa, \pi)}, C$. An $\left(\Omega \in M_{2}\right)$ yields (11)

All other cases are seen easily from IH.

Lemma 5.2 Let $\lambda \leq \pi$ be a regular ordinal term such that $\forall i\left(K\left(m_{i}(\pi)\right) \subset\right.$ $\left.\mathcal{H}_{\max K\left(m_{i}(\pi)\right)}[\Theta]\right)$, and $\Gamma \subset \Sigma_{1}(\lambda)$.

Suppose for an ordinal term $a \in O T$

$$
\left(\mathcal{H}_{\gamma}, \Theta\right) \vdash_{\pi}^{a} \Gamma
$$

where $\{\gamma, \lambda, \pi\} \subset \mathcal{H}_{\gamma}[\Theta]$.

Assume

$$
\forall \rho \in[\lambda, \pi] \forall d\left[\Theta \subset \psi_{\rho}(\gamma \# d)\right]
$$

Let $\hat{a}=\gamma \# \omega^{\pi+a+1}$ and $\beta=\psi_{\lambda}(\hat{a})$. Then the following holds

$$
\left(\mathcal{H}_{\hat{a}+1}, \Theta\right) \vdash_{\beta}^{\beta} \Gamma
$$

Proof by main induction on $\pi$ with subsidiary induction on $a$. We can assume $a>0$.

We see that $\Theta \subset \beta=\psi_{\lambda}(\hat{a})$ from (21). Hence

$$
a_{0} \in \mathcal{H}_{\gamma}[\Theta] \cap a \Rightarrow \psi_{\lambda}\left(\widehat{a_{0}}\right)<\psi_{\lambda}(\hat{a})
$$

Let $\vec{\xi} \in S D$ be a sequence of ordinals and $k$ a number for which the following hold: If $\pi=\mathbb{K}$, then let $\vec{\xi}=\overrightarrow{0}$ with $\operatorname{lh}(\vec{\xi})=N-1$ and $k=N-1$. Let $\pi<\mathbb{K}$. If $\vec{m}(\pi) \neq \overrightarrow{0}$, then $K(\vec{\xi}) \subset \mathcal{H}_{\gamma}[\Theta], \vec{\xi} \leq \vec{m}(\pi)$ and $k=\max \left\{k \leq N-2: \xi_{k+1}>0\right\}$. Otherwise let $\vec{\xi}=\overrightarrow{0}$ and $k=1$. By the assumption (21), and (5) we obtain

$$
\forall \rho \in[\lambda, \pi] \forall b \in K(\vec{\xi}) \forall d\left[\mathrm{k}(\Gamma) \cup\{\gamma, \lambda, a, \pi, b\} \subset \mathcal{H}_{\gamma}\left(\psi_{\rho}(\gamma \# d)\right)\right]
$$


Case 1. First consider the case when $k \geq 2$.

Let $\vec{\xi}=\vec{m}(\pi)$, and $\vec{\zeta}(a):=\left(\zeta_{2}(a), \ldots, \zeta_{k}(a)\right) * \overrightarrow{0}$ be the sequence defined as in Lemma 5.1 from $\gamma, a: \vec{\zeta}(a)=\overrightarrow{0} *(\gamma+a)$ when $\pi=\mathbb{K}$, otherwise $\zeta_{k}(a)=$ $\xi_{k}+\Lambda^{\xi_{k+1}}(\gamma+a)$ and $\zeta_{i}(a)=\xi_{i}$ for $i<k$. Also let $\gamma(a, b)=\gamma \# a \# b$ and $\beta(a, b)=\psi_{\pi} \gamma(a, b)$.

Let $\kappa:=\psi_{\pi}^{\vec{\zeta}(a)}(\gamma(a, 0))$. By the assumption (21) we have $\Theta \subset \psi_{\pi}(\gamma \# a)$. On the other hand we have $\psi_{\pi}(\gamma \# a)=\psi_{\pi}(\gamma(a, 0)) \leq \kappa$, and $\Theta \subset \kappa . \pi \in \mathcal{H}_{\gamma}[\Theta]$ with $\Theta \subset \pi$ yields $K(\vec{\xi})=K(\vec{m}(\pi)) \subset \mathcal{H}_{\gamma}[\Theta] \subset \mathcal{H}_{\gamma(a, 0)}(\kappa)$. Hence $K(\vec{\xi}) \cup$ $\{\pi, \gamma(a, 0)\} \subset \mathcal{H}_{\gamma(a, 0)}(\kappa)$, and $\kappa \in O T$ by $\gamma(a, 0)=\gamma \# a>0$ and Definition 3.12h such that $\kappa<\pi$ and $\mathcal{H}_{\gamma}(\kappa) \cap \pi \subset \kappa$. Moreover we have $\forall i\left(K\left(\zeta_{i}(a)\right) \subset\right.$ $\left.\mathcal{H}_{\max K\left(\zeta_{i}(a)\right)}[\Theta]\right)$ by $\forall i\left(K\left(m_{i}(\pi)\right) \subset \mathcal{H}_{\max K\left(m_{i}(\pi)\right)}[\Theta]\right)$ and $\{\gamma, a\} \subset \mathcal{H}_{\gamma}[\Theta]$ with $\Theta \subset \kappa$. In other words, $\kappa \in H(\vec{\zeta}(a), \pi, \gamma, \Theta)$.

By Lemma 5.1 we obtain $\left(\mathcal{H}_{\gamma(a, \kappa)+1}, \Theta \cup\{\kappa\}\right) \vdash_{\kappa}^{\beta(a, \kappa)} \Gamma^{(\kappa, \pi)}$, and Lemma 4.72 with $\kappa \in \mathcal{H}_{\gamma(a, 0)+1}[\Theta]$

$$
\left(\mathcal{H}_{\gamma(a, \kappa)+1}, \Theta\right) \vdash_{\kappa}^{\beta(a, \kappa)} \Gamma^{(\kappa, \pi)}
$$

If $\lambda=\pi$, then $\Gamma^{(\kappa, \pi)} \subset \Sigma_{1}(\kappa) \subset \Sigma_{0}(\lambda)$. We have $\Theta \subset \psi_{\pi}(\hat{a})=\beta$, and $\kappa \in$ $\mathcal{H}_{\hat{a}}(\beta)$. Hence $\{\gamma, \pi, a, \kappa\} \subset \mathcal{H}_{\hat{a}}(\beta)$, and $\gamma(a, \kappa)=\gamma \# a \# \kappa<\gamma \# \omega^{\pi+a+1}=\hat{a}$. Therefore $\kappa<\beta(a, \kappa) \leq \psi_{\pi}(\hat{a})=\beta$. We obtain (22) by Persistency lemma 4.11

Next consider the case when $\lambda<\pi$. Then $\lambda<\kappa$ and $\Gamma^{(\kappa, \pi)}=\Gamma$. We have for (21), $\forall d \forall \rho \in[\lambda, \kappa]\left(\Theta \subset \psi_{\rho}(\gamma(a, \kappa)+1 \# d)\right)$. By MIH on (24) we obtain $\left(\mathcal{H}_{b_{0}+1}, \Theta\right) \vdash_{\beta_{0}}^{\beta_{0}} \Gamma$ for $\beta_{0}=\psi_{\lambda}\left(b_{0}\right)$ with $b_{0}=(\gamma(a, \kappa)+1) \# \omega^{\kappa+\beta(a, \kappa)+1}$. We have $b_{0}=\gamma \# a \# \kappa \# 1 \# \omega^{\beta(a, \kappa)+1}<\gamma \# \omega^{\pi+a+1}=\hat{a}$ by $\beta(a, \kappa)<\pi$. This yields $\psi_{\lambda}\left(b_{0}\right)=\beta_{0}<\beta=\psi_{\lambda}(\hat{a})$ by $\Theta \subset \beta$ and $\{\gamma, \kappa, \pi, a\} \subset \mathcal{H}_{\hat{a}}(\beta)$. Hence (22) follows.

In what follows suppose $k=1$.

Case 2. Consider the case when the last inference rule is a $(\operatorname{rfl}(\pi, 2, \vec{\xi}, \vec{\nu}))$.

We have an ordinal term $a_{\ell} \in \mathcal{H}_{\gamma}[\Theta] \cap a$, and a finite set $\Delta$ of $\Sigma_{2}(\pi)$-sentences for which $\left(\mathcal{H}_{\gamma}, \Theta\right) \vdash_{\pi}^{a_{\ell}} \Gamma, \neg \delta$ holds for each $\delta \in \Delta$. On the other hand we have sequences $\vec{\nu},\left(\xi_{2}\right) * \overrightarrow{0} \in S D$ such that $\vec{\nu}<_{s d} \xi_{2}$ and $K(\vec{\nu}) \cup K(\vec{\xi}) \subset \mathcal{H}_{\gamma}[\Theta]$ by (7), and an ordinal term $a_{r}(\rho) \in \mathcal{H}_{\gamma}[\Theta \cup\{\rho\}] \cap a$ for which $\left(\mathcal{H}_{\gamma}, \Theta \cup\{\rho\}\right) \vdash_{\pi}^{a_{r}(\rho)}$ $\Gamma, \Delta^{(\rho, \pi)}$ holds for each $\rho \in H(\vec{\nu}, \pi, \gamma, \Theta)$, where $\xi_{2} \leq_{s p} m_{2}(\pi)$.

Let $\rho:=\psi_{\pi}^{\vec{v}}\left(\widehat{a_{\ell}} \# \pi\right)$ for $\widehat{a_{\ell}}=\gamma \# \omega^{\pi+a_{\ell}+1}$. By the assumption (21) we have $\Theta \subset \psi_{\pi}\left(\widehat{a_{\ell}}\right) \subset \rho . K(\vec{\nu}) \cup\{\pi, \gamma, a\} \subset \mathcal{H}_{\gamma}[\Theta]$ yields $K(\vec{\nu}) \cup\left\{\pi, \widehat{a_{\ell}}\right\} \subset \mathcal{H}_{\widehat{a_{\ell}} \# \pi}(\rho)$. Next consider the condition (44). We have $\forall i\left(K\left(\nu_{i}\right) \subset \mathcal{H}_{\max K\left(\nu_{i}\right)}[\Theta]\right)$ by (8), and hence $\forall i\left(K\left(\nu_{i}\right) \subset \mathcal{H}_{\max K\left(\nu_{i}\right)}(\rho)\right)$ by $\Theta \subset \rho$. Therefore $\rho \in O T$ by Definition 3.12i. Moreover $\rho \in C(\pi, \gamma, \Theta)$, i.e., $\mathcal{H}_{\gamma}(\rho) \cap \pi \subset \rho \& \Theta \cap \pi \subset \rho$. Hence $\rho \in H(\vec{\nu}, \pi, \gamma, \Theta)$.

By Inversion lemma 4.9 we obtain for each $\delta \equiv\left(\exists x \in L_{\pi} \delta_{1}(x)\right) \in \Delta$ and each $d \in T m(\rho)$ with $|d|=\max (\{0\} \cup \mathrm{k}(d)),\left(\mathcal{H}_{\gamma \#|d|}, \Theta \cup \mathrm{k}(d)\right) \vdash_{\pi}^{a_{\ell}} \Gamma, \neg \delta_{1}(d)$.

We have $\{\pi, \gamma,|d|\} \subset \mathcal{H}_{\gamma \#|d|}(\pi)$ by $|d|<\rho<\pi$, and this yields $|d| \in$ $\mathcal{H}_{\gamma \#|d|}\left(\psi_{\pi}(\gamma \#|d|)\right) \cap \pi \subset \psi_{\pi}(\gamma \#|d|)$. Hence $|d|<\psi_{\pi}(\gamma \#|d|)$, and $\forall e(\Theta \cup \mathrm{k}(d) \subset$ 
$\psi_{\pi}(\gamma \#|d| \#$ e) $)$, i.e., (21) holds for $\lambda=\pi$ and $\gamma \#|d|$. Let $\beta_{d}=\psi_{\pi}\left(\widehat{a_{d}}\right)$ for $\widehat{a_{d}}=$ $\gamma \#|d| \# \omega^{\pi+a_{\ell}+1}=\widehat{a_{\ell}} \#|d|$. SIH yields $\left(\mathcal{H}_{\widehat{a_{d}}+1}, \Theta \cup \mathrm{k}(d)\right) \vdash_{\beta_{d}}^{\beta_{d}} \Gamma, \neg \delta_{1}(d)$, which in turn Boundedness lemma 4.10 yields $\left(\mathcal{H}_{\widehat{a_{\pi}}+1}, \Theta \cup \mathrm{k}(d)\right) \vdash_{\beta_{d}}^{\beta_{d}} \Gamma, \neg \delta_{1}^{\left(\beta_{d}, \pi\right)}(d)$ for $\widehat{a_{\pi}}=\gamma \# \pi \# \omega^{\pi+a_{\ell}+1}=\widehat{a_{\ell}} \# \pi$. By persistency we obtain $\left(\mathcal{H}_{\widehat{a_{\pi}}+1}, \Theta \cup \mathrm{k}(d)\right) \vdash_{\rho}^{\beta_{d}}$ $\Gamma, \neg \delta_{1}^{(\rho, \pi)}(d)$ for $\beta_{d}<\psi_{\pi}\left(\widehat{a_{\pi}}\right)=\rho \in \mathcal{H}_{\gamma}[\Theta]$. Since $d \in T m(\rho)$ is arbitrary, $(\bigwedge)$ yields

$$
\left(\mathcal{H}_{\widehat{a_{\pi}}+1}, \Theta\right) \vdash_{\rho}^{\rho} \Gamma, \neg \delta^{(\rho, \pi)}
$$

Now pick the $\rho$-th branch from the right upper sequents

$$
\left(\mathcal{H}_{\widehat{a_{\pi}}+1}, \Theta \cup\{\rho\} \vdash_{\pi}^{a_{r}(\rho)} \Gamma, \Delta^{(\rho, \pi)}\right.
$$

By $\rho \in \mathcal{H}_{\widehat{a_{\pi}}+1}[\Theta]$ and Lemma 4.72 we obtain

$$
\left(\mathcal{H}_{\widehat{a_{\pi}}+1}, \Theta\right) \vdash_{\pi}^{a_{r}(\rho)} \Gamma, \Delta^{(\rho, \pi)}
$$

Case 2.1. First consider the case $\lambda=\pi$. Then $\Delta^{(\rho, \pi)} \subset \Sigma_{0}(\lambda)$. Let $\beta_{\rho}=\psi_{\pi}\left(b_{\rho}\right)$ with $b_{\rho}=\widehat{a_{\pi}} \# 1 \# \omega^{\pi+a_{r}(\rho)+1}=\gamma \# \omega^{\pi+a_{\ell}+1} \# \omega^{\pi+a_{r}(\rho)+1} \# \pi \# 1$. Then $\beta_{\rho}>\rho$ and $\forall d\left[\Theta \cup\{\rho\} \subset \psi_{\pi}\left(\widehat{a_{\pi}}+1 \# d\right)\right]$. SIH yields for (26)

$$
\left(\mathcal{H}_{b_{\rho}+1}, \Theta\right) \vdash_{\beta_{\rho}}^{\beta_{\rho}} \Gamma, \Delta^{(\rho, \pi)}
$$

Several (cut)'s with (27), (25) yield $\left(\mathcal{H}_{\hat{a}+1}, \Theta\right) \vdash_{\beta_{\rho}}^{\beta_{\rho}+p} \Gamma$ for $\beta_{\rho} \geq \rho, \widehat{a_{\pi}}<b_{\rho}<\hat{a}$ and some $p<\omega$, where $\beta_{\rho}<\beta=\psi_{\pi}(\hat{a})$ by $b_{\rho}<\hat{a}$. (22) follows.

Case 2.2. Next consider the case when $\lambda<\pi$. Then $\lambda<\rho$ and $\Delta^{(\rho, \pi)} \subset \Sigma_{1}\left(\rho^{+}\right)$ with $\rho^{+}=\Omega_{\rho+1}$. SIH with (26) yields $\left(\mathcal{H}_{b_{\rho}+1}, \Theta \cup\{\rho\}\right) \vdash_{\beta_{\rho^{+}}}^{\beta_{\rho^{+}}} \Gamma, \Delta^{(\rho, \pi)}$ for $\beta_{\rho^{+}}=\psi_{\rho^{+}}\left(b_{\rho}\right)>\rho$, and by Lemma 4.712 we obtain

$$
\left(\mathcal{H}_{b_{\rho}+1}, \Theta\right) \vdash_{\beta_{\rho^{+}}}^{\beta_{\rho^{+}}} \Gamma, \Delta^{(\rho, \pi)}
$$

Several (cut)'s with (25), (28) yield $\left(\mathcal{H}_{b_{0}+1}, \Theta\right) \vdash_{\beta_{\rho^{+}}}^{\beta_{\rho^{+}}+p} \Gamma$ for $\beta_{\rho^{+}}>\rho$ and $b_{0}=\gamma \#\left(\omega^{\pi+a_{\ell}+1} \cdot 2\right) \# \omega^{\pi+a_{r}(\rho)+1} \# 1 \geq \max \left\{b_{\ell}, b_{\rho}\right\}$. Predicative cut-elimination lemma 4.12 yields for $\beta_{1}=\varphi\left(\beta_{\rho^{+}}\right)\left(\beta_{\rho^{+}}+p\right)<\rho^{+}$

$$
\left(\mathcal{H}_{b_{0}+1}, \Theta\right) \vdash_{\rho}^{\beta_{1}} \Gamma
$$

We obtain $\lambda<\rho \in \mathcal{H}_{b_{0}+1}[\Theta]$ by $\gamma<\widehat{a_{\ell}}<b_{0}$. MIH with (29) yields $\left(\mathcal{H}_{c+1}, \Theta\right) \vdash_{\psi_{\lambda} c}^{\psi_{\lambda} c}$ $\Gamma$ for $c=b_{0} \# 1 \# \omega^{\rho+\beta_{1}+1}$. We obtain $c=b_{0} \# \omega^{\rho+\beta_{1}+1} \# 1=\gamma \#\left(\omega^{\pi+a_{\ell}+1}\right.$. 2) $\# \omega^{\pi+a_{r}(\rho)+1} \# \omega^{\rho+\beta_{1}+1} \# 2<\gamma \# \omega^{\pi+a+1}=\hat{a}$ since $a_{\ell}, a_{r}(\rho)<a$ and $\rho, \beta_{1}<$ $\rho^{+}<\pi$. Hence $\psi_{\lambda} c<\psi_{\lambda}(\hat{a})=\beta$, and (22) follows.

Case 3. Third consider the case when the last inference introduces a $\Sigma_{1}(\lambda)$ sentence $(\forall x \in c \theta(x)) \in \Gamma$ for $c \in \operatorname{Tm}(\lambda)$. We have $\left(\mathcal{H}_{\gamma}, \Theta \cup \mathrm{k}(d)\right) \vdash_{\pi}^{a(d)} \Gamma, \theta(d)$ 
for each $d \in \operatorname{Tm}(|c|)$. Then we see from (23) that $|d|<|c| \in \mathcal{H}_{\gamma}\left(\psi_{\rho}(\gamma \# e)\right) \cap \rho \subset$ $\psi_{\rho}(\gamma \# e)$ for any $\rho \in[\lambda, \pi]$ and any $e$. Hence $|d| \in \psi_{\rho}(\gamma \# e)$. (21) is enjoyed for $\Theta \cup \mathrm{k}(d)$. SIH yields $\left(\mathcal{H}_{\hat{a}+1}, \Theta \cup \mathrm{k}(d)\right) \vdash_{\beta_{d}}^{\beta_{d}} \Gamma, \theta(d)$ for $\beta_{d}=\psi_{\lambda}(\widehat{a(d)}) .(\Lambda)$ yields (22) for $\beta=\psi_{\lambda}(\hat{a})>\beta_{d}$.

Case 4. Fourth consider the case when the last inference introduces a $\Sigma_{1}(\lambda)$ sentence $\left(\exists x \in L_{\lambda} \theta(x)\right) \in \Gamma$. We have $\left(\mathcal{H}_{\gamma}, \Theta\right) \vdash_{\pi}^{a_{0}} \Gamma, \theta(d)$ for a $d \in \operatorname{Tm}(\lambda)$. SIH yields $\left(\mathcal{H}_{\hat{a}+1}, \Theta\right) \vdash_{\beta_{0}}^{\beta_{0}} \Gamma, \theta(d)$ for $\beta=\psi_{\lambda}(\hat{a})>\psi_{\lambda}\left(\widehat{a_{0}}\right)=\beta_{0}$. Without loss of generality we can assume that $\mathrm{k}(d) \subset \mathrm{k}(\theta(d))$. Then we see from (23) that $|d| \in \mathcal{H}_{\gamma}\left(\psi_{\lambda}(\gamma+1)\right) \cap \lambda \subset \psi_{\lambda}(\gamma+1)<\beta$. Thus is enjoyed in the following inference rule $(\bigvee)$. We obtain $\left(\mathcal{H}_{\hat{a}+1}, \Theta\right) \vdash_{\beta}^{\beta} \Gamma$ by a $(\bigvee)$, which enjoys ([6).

Case 5. Fifth consider the case when the last inference is a $(\operatorname{rfl}(\tau, j, \vec{\mu}, \vec{\nu}))$ for a $\tau \in \mathcal{H}_{\gamma}[\Theta] \cap \pi$. We have an $a_{\ell}<a$ and a finite set $\Delta$ of $\Sigma_{j}(\tau)$-sentences such that $\left(\mathcal{H}_{\gamma}, \Theta\right) \vdash_{\pi}^{a_{\ell}} \Gamma, \neg \delta$ for each $\delta \in \Delta$. On the other hand we have a sequence $\vec{\nu}$ and an ordinal term $a_{r}(\rho)<a$ for each $\rho \in H(\vec{\nu}, \tau, \gamma, \Theta)$ such that $\left(\mathcal{H}_{\gamma}, \Theta \cup\{\rho\}\right) \vdash_{\pi}^{a_{r}(\rho)} \Gamma, \Delta^{(\rho, \tau)}$. By (23), for any $\rho \in H(\vec{\nu}, \tau, \gamma, \Theta)$ we obtain

$$
\forall e \forall \kappa\left[\max \{\tau+1, \lambda\} \leq \kappa \leq \pi \Rightarrow \rho<\tau \in \mathcal{H}_{\gamma}\left(\psi_{\kappa}(\gamma \# e)\right) \cap \kappa \subset \psi_{\kappa}(\gamma \# e)\right]
$$

Case 5.1. First consider the case when $\tau<\lambda$. Then $\rho<\psi_{\kappa}(\gamma \# e)$ for any $\kappa \in[\lambda, \pi]$ and $e$. From SIH with (30) we obtain $\left(\mathcal{H}_{\hat{a}+1}, \Theta\right) \vdash_{\beta_{\ell}}^{\beta_{\ell}} \Gamma, \neg \delta$ for each $\delta \in \Delta$ with $\beta_{\ell}=\psi_{\lambda}\left(\widehat{a_{\ell}}\right)$, and $\left(\mathcal{H}_{\hat{a}+1}, \Theta \cup\{\rho\}\right) \vdash_{\beta_{r}(\rho)}^{\beta_{r}(\rho)} \Gamma, \Delta{ }^{(\rho, \tau)}$ for each $\rho \in$ $H(\vec{\nu}, \tau, \gamma, \Theta)$ with $\beta_{r}(\rho)=\psi_{\lambda}\left(\widehat{a_{r}(\rho)}\right)$. We see $\max \left\{\beta_{\ell}, \beta_{r}(\rho), \tau\right\}<\beta=\psi_{\lambda}(\hat{a})$, and an inference rule $(\operatorname{rfl}(\tau, j, \vec{\mu}, \vec{\nu}))$ yields $\left(\mathcal{H}_{\hat{a}+1}, \Theta\right) \vdash_{\beta}^{\beta} \Gamma$.

Case 5.2. Second consider the case when $\lambda \leq \tau$. Then $\Delta \cup \Delta^{(\rho, \tau)} \subset \Sigma_{1}\left(\tau^{+}\right)$, and $\rho<\psi_{\kappa}(\gamma \# e)$ for $\tau<\kappa \leq \pi$ and $e$ by (30). SIH yields $\left(\mathcal{H}_{\widehat{a_{\ell}}+1}, \Theta\right) \vdash_{\beta_{2}}^{\beta_{2}} \Gamma, \neg \delta$ for each $\delta \in \Delta$, where $\beta_{2}=\psi_{\tau^{+}}\left(\widehat{a_{\ell}}\right)$. On the other side $\operatorname{SIH}$ yields $\left(\mathcal{H}_{\widehat{a_{r}(\rho)}+1}, \Theta \cup\right.$ $\{\rho\}) \vdash_{\beta_{\rho}}^{\beta_{\rho}} \Gamma, \Delta^{(\rho, \tau)}$ for each $\rho \in H(\vec{\nu}, \tau, \gamma, \Theta)$, where $\beta_{\rho}=\psi_{\tau^{+}}\left(\widehat{a_{r}(\rho)}\right)$. Predicative cut-elimination lemma 4.12 yields $\left(\mathcal{H}_{\widehat{a_{\ell}}+1}, \Theta\right) \vdash_{\tau}^{\delta_{2}} \Gamma, \neg \delta$ and $\left(\mathcal{H}_{\widehat{a_{r}(\rho)}+1}, \Theta \cup\right.$ $\{\rho\}) \vdash_{\tau}^{\delta_{\rho}} \Gamma, \Delta^{(\rho, \tau)}$ for $\delta_{2}=\varphi\left(\beta_{2}\right)\left(\beta_{2}\right)$ and $\delta_{\rho}=\varphi\left(\beta_{\rho}\right)\left(\beta_{\rho}\right)$. From these with the inference rule $(\operatorname{rfl}(\tau, j, \vec{\mu}, \vec{\nu}))$ we obtain

$$
\left(\mathcal{H}_{\widehat{a_{0}}+1}, \Theta\right) \vdash_{\tau}^{\delta_{0}+1} \Gamma
$$

where $\sup \left\{\delta_{2}, \delta_{\rho}: \rho \in H\left(\vec{\nu}, \tau, \widehat{a_{0}}+1, \Theta\right)\right\} \leq \delta_{0}:=\varphi\left(\beta_{0}\right)\left(\beta_{0}\right) \in \mathcal{H}_{\widehat{a_{0}}+1}[\Theta]$ with $\sup \left\{\beta_{2}, \beta_{\rho}: \rho \in H(\vec{\nu}, \tau, \gamma, \Theta)\right\} \leq \beta_{0}:=\psi_{\tau^{+}}\left(\widehat{a_{0}}\right)$, and $\sup \left\{a_{\ell}, a_{r}(\rho): \rho \in\right.$ $H(\vec{\nu}, \tau, \gamma, \Theta)\} \leq a_{0} \in \mathcal{H}_{\gamma}[\Theta] \cap a$, cf. (10).

MIH with (31) yields $\left(\mathcal{H}_{\hat{a}+1}, \Theta\right) \vdash_{\delta}^{\delta} \Gamma$ for $\delta=\psi_{\lambda}\left(\left(\widehat{a_{0}}+1\right) \# \omega^{\tau+\delta_{0}+2}\right)$ and $\left(\widehat{a_{0}}+1\right) \# \omega^{\tau+\delta_{0}+2}<\hat{a}$. We have $\delta=\psi_{\lambda}\left(\widehat{a_{0}} \# 1 \# \omega^{\tau+\delta_{0}+2}\right)<\psi_{\lambda}(\hat{a})=\beta$ by $\widehat{a_{0}}<\hat{a}$ and $\tau, \delta_{0}<\tau^{+}<\pi$ and $\tau \in \mathcal{H}_{\gamma}[\Theta]$. (22) follows. 
Case 6. Sixth consider the case when the last inference is a (cut). For an $a_{0}<a$ and a $C$ with $\operatorname{rk}(C)<\pi$, we have $\left(\mathcal{H}_{\gamma}, \Theta\right) \vdash_{\pi}^{a_{0}} \Gamma, \neg C$ and $\left(\mathcal{H}_{\gamma}, \Theta\right) \vdash_{\pi}^{a_{0}} C, \Gamma$.

Case 6.1. First consider the case when $\operatorname{rk}(C)<\lambda$. Then $C \in \Sigma_{0}(\lambda)$. SIH yields the lemma.

Case 6.2. Second consider the case when $\lambda \leq \operatorname{rk}(C)<\pi$. Let $\rho^{+}=(\operatorname{rk}(C))^{+}=$ $\min \{\kappa \in \operatorname{Reg}: \operatorname{rk}(C)<\kappa\}$. Then $C \in \Sigma_{0}\left(\rho^{+}\right)$and $\lambda \leq \rho \in \mathcal{H}_{\gamma}[\Theta] \cap \pi$. SIH yields $\left(\mathcal{H}_{\widehat{a_{0}}+1}, \Theta\right) \vdash_{\beta_{0}}^{\beta_{0}} \Gamma, \neg C$ and $\left(\mathcal{H}_{\widehat{a_{0}}+1}, \Theta\right) \vdash_{\beta_{0}}^{\beta_{0}} C, \Gamma$ for $\beta_{0}=\psi_{\rho^{+}}\left(\widehat{a_{0}}\right) \in \mathcal{H}_{\widehat{a_{0}}+1}[\Theta]$. By a $($ cut $)$ we obtain $\left(\mathcal{H}_{\widehat{a_{0}}+1}, \Theta\right) \vdash_{\beta_{1}}^{\beta_{1}} \Gamma$ for $\beta_{1}=\max \left\{\beta_{0}, \operatorname{rk}(C)\right\}+1$ with $\rho<$ $\beta_{1}<\rho^{+}$. Predicative cut-elimination lemma 4.12 yields $\left(\mathcal{H}_{\widehat{a_{0}}+1}, \Theta\right) \vdash_{\rho}^{\delta_{1}} \Gamma$ for $\delta_{1}=\varphi\left(\beta_{1}\right)\left(\beta_{1}\right)$, where $\widehat{a_{0}} \in \mathcal{H}_{\widehat{a_{0}}+1}[\Theta]$, and $\forall e \forall \tau \in[\lambda, \rho]\left[\Theta \subset \psi_{\tau}\left(\widehat{a_{0}} \# e\right)\right]$ hold. Hence MIH with $\rho \in \mathcal{H}_{\widehat{a_{0}}+1}[\Theta]$ yields $\left(\mathcal{H}_{b+1}, \Theta\right) \vdash_{\psi_{\lambda}(b)}^{\psi_{\lambda}(b)} \Gamma$ for $b=\widehat{a_{0}} \# 1 \# \omega^{\rho+\delta_{1}+1}$. We see $b<\hat{a}$ and $\psi_{\lambda}(b)<\psi_{\lambda}(\hat{a})=\beta$, and (22) follows.

Case 7. Seventh consider the case when the last inference is an $\left(\Omega \in M_{2}\right)$. We have $\left(\mathcal{H}_{\gamma}, \Theta\right) \vdash_{\pi}^{a_{\ell}} \Gamma, C$ for an $a_{\ell}<a$, and $\left(\mathcal{H}_{\gamma}, \Theta \cup\{\alpha\}\right) \vdash_{\pi}^{a_{r}(\alpha)} \neg C^{(\alpha, \Omega)}, \Gamma$ for an $a_{r}(\alpha)<a$ for each $\alpha<\Omega$, where $C \in \Pi_{2}(\Omega)$.

The case $\lambda>\Omega$ is seen as in Case 5.1. The case $\lambda=\Omega$ is seen as in Case 5.2 .

Let us conclude Theorem 1.1 Let $\Omega=\Omega_{1}$.

Proof of Theorem 1.1] Let $\mathrm{KP} \Pi_{N} \vdash \theta$. By Embedding lemma 4.14 pick an $m$ so that $\left(\mathcal{H}_{0}, \emptyset\right) \vdash \vdash_{\mathbb{K}+m}^{\mathbb{K} \cdot 2+m} \theta$. Predicative cut-elimination lemma 4.12 yields $\left(\mathcal{H}_{0}, \emptyset\right) \vdash_{\mathbb{K}}^{\omega_{m+1}(\mathbb{K}+1)} \theta$ for $\omega_{m}(\mathbb{K} \cdot 2+m)<\omega_{m+1}(\mathbb{K}+1)$. Lemma 5.2 yields $\left(\mathcal{H}_{a+1}, \emptyset\right) \vdash_{\beta}^{\beta} \theta$ for $a=\omega^{\mathbb{K}+\omega_{m+1}(\mathbb{K}+1)+1}$ and $\beta=\psi_{\Omega}(a)$. Predicative cutelimination lemma 4.12 yields $\left(\mathcal{H}_{a+1}, \emptyset\right) \vdash_{0}^{\varphi(\beta)(\beta)} \theta$. We obtain $\varphi(\beta)(\beta)<\alpha:=$ $\psi_{\Omega}\left(\omega_{n}(\mathbb{K}+1)\right)$ for $n=m+3$, and hence $\left(\mathcal{H}_{\omega_{n}(\mathbb{K}+1)}, \emptyset\right) \vdash_{0}^{\alpha} \theta$. Boundedness lemma 4.10 yields $\left(\mathcal{H}_{\omega_{n}(\mathbb{K}+1)}, \emptyset\right) \vdash_{0}^{\alpha} \theta^{(\alpha, \Omega)}$. Since each inference rule other than reflection rules $(\operatorname{rfl}(\pi, k, \vec{\xi}, \vec{\nu}))$ and $\left(\Omega \in M_{2}\right)$ is sound, we see by induction up to $\alpha=\psi_{\Omega}\left(\omega_{n}(\mathbb{K}+1)\right)$ that $L_{\alpha}=\theta$.

This completes a proof of Theorem 1.1 .

\section{References}

[1] T. Arai, Iterating the recursively Mahlo operations, in Proceedings of the thirteenth International Congress of Logic Methodology, Philosophy of Science, eds. C. Glymour, W. Wei and D. Westerstahl (College Publications, King's College, London, 2009), pp. 21-35. 
[2] T. Arai, Wellfoundedness proofs by means of non-monotonic inductive definitions II: first order operators, Ann. Pure Appl. Logic 162 (2010), pp. 107-143.

[3] T. Arai, Conservations of first-order reflections, Jour. Symb. Logic 79 (2014), pp. 814-825.

[4] T. Arai, Proof theory for theories of ordinals III: $\Pi_{N}$-reflection, Gentzen's centenary: the quest of consistency, eds. R. Kahle and M. Rathjen, pp. 357-424, Springer, 2015.

[5] T. Arai, Wellfoundedness proof for first-order reflection, draft. posted to the arxiv.

[6] W. Buchholz, A simplified version of local predicativity, in Proof Theory, eds. P. H. G. Aczel, H. Simmons and S. S. Wainer (Cambridge UP,1992), pp. $115-147$.

[7] W. Pohlers and J.-C. Stegert, Provable recursive functions of reflection, in Logic, Construction, Computation, eds. U. Berger, H. Diener, P. Schuster and M. Seisenberger, Ontos Mathematical Logic vol. 3 (De Gruyter, 2012), pp. 381-474.

[8] M. Rathjen, Proof theory of reflection, Ann. Pure Appl. Logic 68 (1994) $181-224$.

[9] M. Rathjen, An ordinal analysis of stability, Arch. Math. Logic 44 (2005) 1-62. 